\title{
Diphoton resonance confronts dark matter
}

\author{
Soo-Min Choi, Yoo-Jin Kang and Hyun Min Lee \\ Department of Physics, Chung-Ang University, \\ 06974 Seoul, Korea \\ E-mail: sm90515@cau.ac.kr, dbwlsdl1008@cau.ac.kr, hminlee@cau.ac.kr
}

ABSTRACT: As an interpretation of the $750 \mathrm{GeV}$ diphoton excesses recently reported by both ATLAS and CMS collaborations, we consider a simple extension of the Standard Model with a Dirac fermion dark matter where a singlet complex scalar field mediates between dark matter and SM particles via effective couplings to SM gauge bosons and/or Higgs-portal. In this model, we can accommodate the diphoton events through the direct and/or cascade decays of pseudo-scalar and real scalar partners of the complex scalar field. We show that mono-jet searches and gamma-ray observations are complementary in constraining the region where the width of the diphoton resonance can be enhanced due to the couplings of the resonance to dark matter and the correct relic density is obtained. In the case of cascade decay of the resonance, the effective couplings of singlet scalars can be smaller, but the model is still testable by the future discrimination between single photon and photon-jet at the LHC as well as the gamma-ray searches for the cascade annihilation of dark matter.

KEywords: Beyond Standard Model, Cosmology of Theories beyond the SM, Global Symmetries

ARXIV EPRINT: 1605.04804 


\section{Contents}

1 Introduction 1

2 The model 3

3 Diphoton resonance at the LHC 5

3.1 Diphotons from direct decay 5

$\begin{array}{lll}3.2 & \text { Diphotons from cascade decay } & 8\end{array}$

4 Dark matter with pseudo-scalar resonance $\quad 12$

$\begin{array}{ll}\text { 4.1 Dark matter annihilation } & 12\end{array}$

$\begin{array}{ll}4.2 \text { Bounds from indirect detections } & 14\end{array}$

5 Dark matter with real-scalar resonance $\quad 15$

6 Dark matter with pseudo- and real-scalars 18

$\begin{array}{lll}6.1 & \text { Dark matter annihilation } & 18\end{array}$

$\begin{array}{ll}6.2 \text { Indirect detection } & 19\end{array}$

$\begin{array}{lll}7 & \text { Conclusions } & 22\end{array}$

$\begin{array}{ll}\text { A Scalar sector of the model } & 23\end{array}$

\section{Introduction}

Recently there have been tantalizing hints for new physics from the diphoton excesses at about $750 \mathrm{GeV}$ with local significances of $3.9 \sigma$ and $2.6 \sigma$, that have been observed in LHC Run 1 data at $13 \mathrm{TeV}$ by both ATLAS and CMS collaborations [1, 2], respectively. After Moriond 2016 conference, the results are updated with $8 \mathrm{TeV}$ data included in the analysis $[3,4]$, leading to higher significances, in particular, to $3.4 \sigma$ in the case of CMS. ATLAS favors a wide width of the resonance about $45 \mathrm{GeV}$, but the significance changes only by $0.3 \sigma$ as compared to the case with narrow width. Furthermore, CMS prefers a narrow width in their best fit result. The production cross section required for explaining the diphoton excesses is about $6 \mathrm{fb}$, although the result depends on the assumption of the resonance width [5-11]. The production cross section for the diphoton resonance appears relatively large for given collider bounds from other related LHC searches $[5,6]$. The typical interpretation of diphoton excesses with a new scalar resonance calls for extra vector-like fermions with sizable Yukawa couplings to the resonance $[5,6,12-29]$. In the case of a spin-2 resonance such as Kaluza-Klein graviton, a nontrivial positioning of SM particles in 
extra dimensions is necessary to satisfy strong bounds from electroweak precision data and dilepton and di-jet searches [30-34]. Unitarity arguments on the resonance might imply the coexistence of scalar and extra resonances of higher spin in QCD-like theories or gravity duals in extra dimensions $[35,36]$.

At the moment, we don't have enough information to tell about the properties of the resonance such as width and spin/parity, but we will be able to know them from LHC Run 2 data at $13 \mathrm{TeV}$. In the mean time, it would be interesting to entertain the possibility of a sizable width scenario that can be consistent with the diphoton excesses and other experimental bounds. If the invisible decay mode of the resonance, which is less constrained, is responsible for a large width of the resonance, there is an interesting possibility that the resonance plays a role of mediator between the SM and dark matter [30, 37-49]. On the other hand, there is a plausible option to explain the diphoton excesses with collimated photons, the so called photon-jets, which come from a cascade decay of the resonance into a pair of light mediators, each of them decaying into a pair of photons [50-57]. In this case, the width of the resonance can be increased by a renormalizable coupling between the resonance and the light mediator.

In this article, we consider a simple extension of the SM with a complex singlet scalar field that couples to both the SM and Dirac fermion dark matter in the presence of an approximate $\mathrm{U}(1)$ global symmetry. A soft breaking of the global symmetry induces a nonzero mass for the would-be Goldstone boson or pseudo-scalar, so the model is consistent with phenomenological bounds. We introduce effective couplings of real-scalar and pseudoscalar of the complex scalar field to the SM gauge bosons as a consequence of integrating out new vector-like fermions and the real-scalar can also couple to the SM particles just like the SM Higgs via Higgs-portal. The U(1) invariant couplings of the complex scalar field to vector-like fermions fix the ratio of effective couplings of real-scalar and pseudo-scalar in our model.

We identify the real-scalar and/or pseudo-scalar as the diphoton resonance in our model and consider the possibilities of explaining the diphoton excesses in terms of the direct and/or cascade decays of the resonance. In each case, we impose the collider bounds such as mono-jet and di-jet bounds as well as indirect bounds from gamma-ray and antiproton searches for dark matter. As illustrated from benchmark models that satisfy all the phenomenological constraints, we show that there is an interesting interplay between mono-jet and gamma-ray searches in the case of direct decay whereas those bounds can be weakened in the case of cascade decay due to smaller effective couplings of the singlet scalars. In the latter case, the discrimination between single photon and photon-jet in the LHC Run 2 would become more important. On the side of cosmic data, the same coupling responsible for the cascade decay of the resonance leads to the cascade annihilation of dark matter into multiple photons leading to interesting signatures such as gamma-ray box.

This paper is organized as follows. We begin with a description for the interactions of singlet scalars in our model and present the necessary formulas for the partial decay rates. Then, we discuss the diphoton conditions in the cases of direct and/or cascade decays of the singlet scalar(s) and constrain the parameter space of effective couplings of the resonances. Next we consider the annihilation of dark matter with the singlet scalar mediators in each 
scenario of the diphoton interpretation and show how collider and cosmic data can be used to constrain the models. There is an appendix containing the scalar potential and scalar self-interactions in our model. Finally, conclusions are drawn.

\section{The model}

We consider a complex singlet scalar $S$ and a Dirac fermion dark matter $\chi$, that transform under a U(1) global symmetry as $S \rightarrow e^{-2 i \alpha} S$ and $\chi \rightarrow e^{i \gamma_{5} \alpha} \chi$, respectively. Expanding the complex scalar $S$ around a VEV as in the appendix and integrating out vector-like fermions [58-61], we obtain the following effective Lagrangian for a singlet pseudo-scalar $a$, two CP-even scalars, Higgs-like $h_{1}$ and singlet-like $h_{2}$, and dark matter,

$$
\begin{gathered}
\mathcal{L}=\bar{\chi}\left(i \gamma^{\mu} \partial_{\mu}-m_{\chi}\right) \chi+\frac{1}{2}\left(\partial_{\mu} a\right)^{2}-\frac{1}{2} m_{a}^{2} a^{2}+\sum_{i=1,2}\left(\frac{1}{2}\left(\partial_{\mu} h_{i}\right)^{2}-\frac{1}{2} m_{i}^{2} h_{i}^{2}\right) \\
+\frac{1}{\sqrt{2}} i \lambda_{\chi} a \bar{\chi} \gamma^{5} \chi-\frac{1}{\sqrt{2}} \lambda_{\chi}\left(h_{2} \cos \theta+h_{1} \sin \theta\right) \bar{\chi} \chi+\mathcal{L}_{\text {scalar }}+\mathcal{L}_{\mathrm{D} 5}
\end{gathered}
$$

where $\mathcal{L}_{\text {scalar }}$ is the interaction Lagrangian for scalars only given in eq. (A.7) and $\mathcal{L}_{D 5}$ contains the dimension- 5 interactions of singlet scalars to gauge fields, given by

$$
\begin{aligned}
\mathcal{L}_{\mathrm{D} 5}= & \frac{1}{\Lambda} a\left(c_{1} F_{\mu \nu}^{Y} \tilde{F}^{Y \mu \nu}+c_{2} W_{\mu \nu} \tilde{W}^{\mu \nu}+c_{3} G_{\mu \nu} \tilde{G}^{\mu \nu}\right) \\
& +\frac{1}{\Lambda} h_{2} \cos \theta\left(d_{1} F_{\mu \nu}^{Y} F^{Y \mu \nu}+d_{2} W_{\mu \nu} W^{\mu \nu}+d_{3} G_{\mu \nu} G^{\mu \nu}\right) \\
& +\frac{1}{\Lambda} h_{1} \sin \theta\left(\hat{d}_{1} F_{\mu \nu}^{Y} F^{Y \mu \nu}+\hat{d}_{2} W_{\mu \nu} W^{\mu \nu}+\hat{d}_{3} G_{\mu \nu} G^{\mu \nu}\right)
\end{aligned}
$$

with the dual field strength tensor being $\tilde{F}_{\mu \nu} \equiv \frac{1}{2} \epsilon_{\mu \nu \rho \sigma} F^{\rho \sigma}$, etc, and $c_{i}, d_{i}, \hat{d}_{i}(i=1,2,3)$ being effective couplings induced by vector-like fermions. We note that when vector-like fermion have the same global charges as for dark matter $[58,59]$, in the decoupling limit of vector-like fermions, the effective couplings are related by $d_{i}=\frac{4}{3} c_{i}(i=1,2,3)$. Then, singlet scalars communicate between dark matter and the SM particles, via effective gauge couplings and Higgs-portal. Similar models [58-60, 62] have been considered in light of the Fermi-LAT gamma-ray line, satisfying various bounds from indirect and direct detections as well as collider experiments. The interplay between dark matter detection and collider experiments in the cases with vector [60] or tensor [66, 67] mediators have been also discussed in the previous works.

In our model, the $\mathrm{U}(1)$ global symmetry is broken to a $Z_{2}$ discrete symmetry, which ensures the stability of dark matter fermion. Any global charges are vulnerable to quantum gravity effects, ${ }^{1}$ but the violation of a global symmetry could be ensured at sufficiently higher orders of effective interactions in the presence of extra discrete gauge symmetries $[64,65]$. We assume that this is the case without changing the low-energy phenomenology.

\footnotetext{
${ }^{1}$ See a recent discussion on the classification of effective interactions that violate the global symmetries of dark matter [63].
} 
In the basis of physical gauge bosons, the loop-induced couplings in eq. (2.2) can be rewritten as

$$
\begin{aligned}
\mathcal{L}_{\mathrm{D} 5}= & \frac{1}{\Lambda} a\left(c_{\gamma \gamma} F_{\mu \nu} \tilde{F}^{\mu \nu}+c_{\gamma Z} F_{\mu \nu} \tilde{Z}^{\mu \nu}+c_{W W} W_{\mu \nu}^{+} \tilde{W}^{-\mu \nu}+c_{Z Z} Z_{\mu \nu} \tilde{Z}^{\mu \nu}+c_{g g} G_{\mu \nu} \tilde{G}^{\mu \nu}\right) \\
& +\frac{1}{\Lambda} h_{2}\left(d_{\gamma \gamma} F_{\mu \nu} F^{\mu \nu}+d_{\gamma Z} F_{\mu \nu} Z^{\mu \nu}+d_{W W} W_{\mu \nu}^{+} W^{-\mu \nu}\right. \\
& \left.+d_{Z Z} Z_{\mu \nu} Z^{\mu \nu}+d_{g g} G_{\mu \nu} G^{\mu \nu}\right) \\
& +\frac{1}{\Lambda} h_{1}\left(\hat{d}_{\gamma \gamma} F_{\mu \nu} F^{\mu \nu}+\hat{d}_{\gamma Z} F_{\mu \nu} Z^{\mu \nu}+\hat{d}_{W W} W_{\mu \nu}^{+} W^{-\mu \nu}\right. \\
& \left.+\hat{d}_{Z Z} Z_{\mu \nu} Z^{\mu \nu}+\hat{d}_{g g} G_{\mu \nu} G^{\mu \nu}\right)
\end{aligned}
$$

with

$$
\begin{aligned}
c_{\gamma \gamma} & =c_{1} \cos ^{2} \theta_{W}+c_{2} \sin ^{2} \theta_{W}, \quad c_{\gamma Z}=\left(c_{2}-c_{1}\right) \sin \left(2 \theta_{W}\right), \\
c_{W W} & =2 c_{2} . \quad c_{Z Z}=c_{1} \sin ^{2} \theta_{W}+c_{2} \cos ^{2} \theta_{W}, \quad c_{g g}=c_{3}, \\
d_{\gamma \gamma} & =\left(d_{1} \cos ^{2} \theta_{W}+d_{2} \sin ^{2} \theta_{W}\right) \cos \theta, \quad d_{\gamma Z}=\left(d_{2}-d_{1}\right) \sin \left(2 \theta_{W}\right) \cos \theta, \\
d_{W W} & =2 d_{2} \cos \theta . \quad d_{Z Z}=\left(d_{1} \sin ^{2} \theta_{W}+d_{2} \cos ^{2} \theta_{W}\right) \cos \theta, \quad d_{g g}=d_{3} \cos \theta,
\end{aligned}
$$

and

$$
\begin{aligned}
\hat{d}_{\gamma \gamma} & =\left(\hat{d}_{1} \cos ^{2} \theta_{W}+\hat{d}_{2} \sin ^{2} \theta_{W}\right) \sin \theta, \quad \hat{d}_{\gamma Z}=\left(\hat{d}_{2}-\hat{d}_{1}\right) \sin \left(2 \theta_{W}\right) \sin \theta, \\
\hat{d}_{W W} & =2 \hat{d}_{2} \sin \theta . \quad \hat{d}_{Z Z}=\left(\hat{d}_{1} \sin ^{2} \theta_{W}+\hat{d}_{2} \cos ^{2} \theta_{W}\right) \sin \theta, \quad \hat{d}_{g g}=\hat{d}_{3} \sin \theta .
\end{aligned}
$$

The total decay rate of the pseudo-scalar is given by $\Gamma_{a}=\sum_{i} \Gamma_{a}(i)$, with the partial decay rates of the pseudo-scalar being

$$
\begin{aligned}
\Gamma_{a}(g g) & =\frac{2 m_{a}^{3}}{\pi \Lambda^{2}} c_{g g}^{2} \\
\Gamma_{a}(\gamma \gamma) & =\frac{m_{a}^{3}}{4 \pi \Lambda^{2}} c_{\gamma \gamma}^{2} \\
\Gamma_{a}(Z \gamma) & =\frac{m_{a}^{3}}{8 \pi \Lambda^{2}} c_{Z \gamma}^{2}\left(1-\frac{m_{Z}^{2}}{m_{a}^{2}}\right)^{3}, \\
\Gamma_{a}(Z Z) & =\frac{m_{a}^{3}}{4 \pi \Lambda^{2}} c_{Z Z}^{2}\left(1-\frac{4 m_{Z}^{2}}{m_{a}^{2}}\right)^{3 / 2}, \\
\Gamma_{a}(W W) & =\frac{m_{a}^{3}}{8 \pi \Lambda^{2}} c_{W W}^{2}\left(1-\frac{4 m_{W}^{2}}{m_{a}^{2}}\right)^{3 / 2} \\
\Gamma_{a}(\bar{\chi} \chi) & =\frac{\lambda_{\chi}^{2} m_{a}}{16 \pi}\left(1-\frac{4 m_{\chi}^{2}}{m_{a}^{2}}\right)^{1 / 2} .
\end{aligned}
$$

The case with a nonzero Higgs mixing angle is potentially interesting for the exotic decays of the SM Higgs boson. However, given the strong limits from Higgs data [68-71], we focus on the case with a negligibly small Higgs mixing, $\sin \theta \sim 0$, so that there is no modification in the couplings of the SM Higgs. For $\sin \theta \sim 0$, the total decay rate 

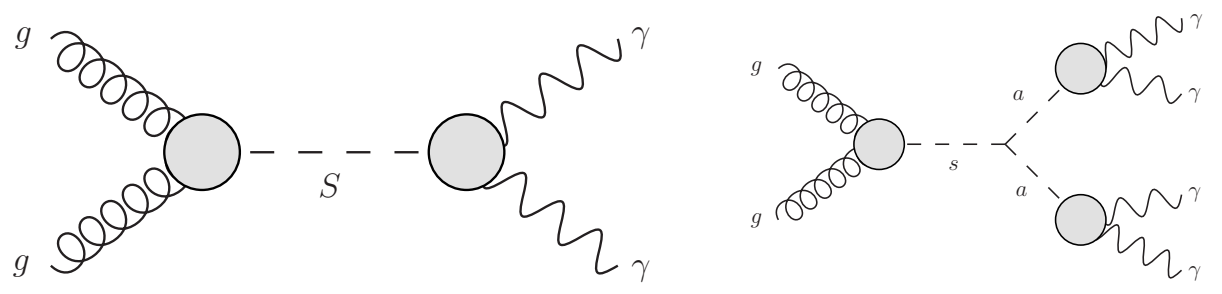

Figure 1. Feynman diagrams for diphoton production with gluon fusion.

of the singlet-like scalar is given by $\Gamma_{2}=\sum_{i} \Gamma_{h_{2}}(i)$, with the partial decay rates of the pseudo-scalar being

$$
\begin{aligned}
\Gamma_{2}(g g) & =\frac{2 m_{2}^{3}}{\pi \Lambda^{2}} d_{g g}^{2} \\
\Gamma_{2}(\gamma \gamma) & =\frac{m_{2}^{3}}{4 \pi \Lambda^{2}} d_{\gamma \gamma}^{2} \\
\Gamma_{2}(Z \gamma) & =\frac{m_{2}^{3}}{8 \pi \Lambda^{2}} d_{Z \gamma}^{2}\left(1-\frac{m_{Z}^{2}}{m_{2}^{2}}\right)^{3} \\
\Gamma_{2}(Z Z) & =\frac{m_{2}^{3}}{4 \pi \Lambda^{2}} d_{Z Z}^{2}\left(1-\frac{4 m_{Z}^{2}}{m_{2}^{2}}+\frac{6 m_{Z}^{4}}{m_{2}^{4}}\right)\left(1-\frac{4 m_{Z}^{2}}{m_{2}^{2}}\right)^{1 / 2}, \\
\Gamma_{2}(W W) & =\frac{m_{2}^{3}}{8 \pi \Lambda^{2}} d_{W W}^{2}\left(1-\frac{4 m_{W}^{2}}{m_{2}^{2}}+\frac{6 m_{W}^{4}}{m_{2}^{4}}\right)\left(1-\frac{4 m_{W}^{2}}{m_{2}^{2}}\right)^{1 / 2} \\
\Gamma_{2}(\bar{\chi} \chi) & =\frac{\lambda_{\chi}^{2} m_{2}}{16 \pi}\left(1-\frac{4 m_{\chi}^{2}}{m_{2}^{2}}\right)^{3 / 2}, \\
\Gamma_{2}(a a) & =\frac{\lambda_{S}^{2} v_{s}^{2}}{8 \pi m_{2}}\left(1-\frac{4 m_{a}^{2}}{m_{2}^{2}}\right)^{1 / 2} .
\end{aligned}
$$

Here, in the $a a$ decay mode, $\lambda_{S}$ is the quartic coupling for the complex scalar field $S$, as introduced in the appendix.

\section{Diphoton resonance at the $\mathrm{LHC}$}

The recently observed diphoton excess near $750 \mathrm{GeV}$ can be explained by the direct decay of a new neutral resonance beyond the SM. Nonetheless, there are more possibilities to explain the diphoton resonance with the direct decays of two degenerate resonances or with a cascade decay of the resonance into multi-photons through light intermediate states as shown in figure 1. In this section, we consider each of the possibilities in the model and constrain the effective couplings of the resonances.

\subsection{Diphotons from direct decay}

The resonance production cross section of scalar particle(s) $X$ via gluon fusion followed by its diphoton decay at the LHC is given $[5,6]$ by

$$
\sigma(p p \rightarrow X \rightarrow \gamma \gamma)=\frac{1}{s M_{X} \Gamma_{X}} K_{g g} C_{g g} \Gamma(X \rightarrow g g) \Gamma(X \rightarrow \gamma \gamma)
$$


where the gluon luminosity is given by $C_{g g}=2137$ for $s=(13 \mathrm{TeV})^{2}$ and the $K$-factor is given by $K_{g g}=1.5$. In the case with resonances from pseudo-scalar $a$ and/or real scalar $s$, we have $X=a$ and/or $s$.

When there is only one resonance due to either pseudo-scalar or real scalar, i.e., $X=a$ or $s$, the diphoton production cross section leads to

$$
\Gamma(X \rightarrow g g) \Gamma(X \rightarrow \gamma \gamma)=2.8 \times 10^{-2} \mathrm{GeV}^{2}\left(\frac{\sigma(p p \rightarrow \gamma \gamma)}{6 \mathrm{fb}}\right)\left(\frac{\Gamma_{X}}{45 \mathrm{GeV}}\right)
$$

For $X=a$, from eqs. (2.11) and (2.12), we get the condition on the effective couplings of the resonance as

$$
\left|c_{g g} \cdot c_{\gamma \gamma}\right|=0.016\left(\frac{\sigma(p p \rightarrow \gamma \gamma)}{6 \mathrm{fb}}\right)^{1 / 2}\left(\frac{\Lambda}{3 \mathrm{TeV}}\right)^{2}\left(\frac{\Gamma_{a}}{45 \mathrm{GeV}}\right)^{1 / 2} .
$$

The $g g$ and $\gamma \gamma$ modes only do not tend to give rise to a wide width due to di-jet bound, unless the photon coupling to the resonance is large. Therefore, if the wide width of the resonance is necessary, one has to rely on other decay modes of the resonance, such as the invisible decay mode into a pair of dark matter particles. In figure 2, we depict the parameter space for the effective gluon and photon couplings of the pseudo-scalar field, explaining the diphoton excess and satisfying the di-jet bound as well as the mono-jet bound in the presence of the invisible decay mode. We have set $c_{2}=0$ and the diphoton production cross section of $\sigma(p p \rightarrow \gamma \gamma)=6 \pm 3 \mathrm{fb}$ is imposed. As we increase the invisible decay rate, the mono-jet bound becomes more sensitive to rule out a sizable gluon coupling.

For $X=h_{2}$, there is a similar condition for the diphoton resonance, with $c_{g g}, c_{\gamma \gamma}$ being replaced by $d_{g g}, d_{\gamma \gamma}$, respectively, so there are similar limits from mono-jet and di-jet searches as those obtained for the pseudo-scalar resonance in the later discussion.

When there are two resonances with $m_{a} \approx m_{2} \approx 750 \mathrm{GeV}$, namely, $X=a$ and $h_{2}$, two singlet scalars contribute to the diphoton excesses, with the diphoton production cross section being constrained by

$$
\Gamma_{a}(g g) \Gamma_{a}(\gamma \gamma)+\frac{\Gamma_{a}}{\Gamma_{2}} \Gamma_{2}(g g) \Gamma_{2}(\gamma \gamma)=2.8 \times 10^{-2} \mathrm{GeV}^{2}\left(\frac{\sigma(p p \rightarrow \gamma \gamma)}{6 \mathrm{fb}}\right)\left(\frac{\Gamma_{a}}{45 \mathrm{GeV}}\right) .
$$

Then, from eqs. (2.11), (2.12), (2.17) and (2.18), the effective couplings of the resonances are constrained to

$$
\sqrt{c_{g g}^{2} c_{\gamma \gamma}^{2}+\frac{\Gamma_{a}}{\Gamma_{2}} d_{g g}^{2} d_{\gamma \gamma}^{2}}=0.016\left(\frac{\sigma(p p \rightarrow \gamma \gamma)}{6 \mathrm{fb}}\right)^{1 / 2}\left(\frac{\Lambda}{3 \mathrm{TeV}}\right)^{2}\left(\frac{\Gamma_{a}}{45 \mathrm{GeV}}\right)^{1 / 2} .
$$

In the decoupling limit of vector-like fermions that have the same global charges as dark matter, we get $d_{g g}=\frac{4}{3} c_{g g}$ and $d_{\gamma \gamma}=\frac{4}{3} c_{\gamma \gamma}$ again so the above condition becomes

$$
\left|c_{g g} \cdot c_{\gamma \gamma}\right| \sqrt{1+\frac{256 \Gamma_{a}}{81 \Gamma_{2}}}=0.016\left(\frac{\sigma(p p \rightarrow \gamma \gamma)}{6 \mathrm{fb}}\right)^{1 / 2}\left(\frac{\Lambda}{3 \mathrm{TeV}}\right)^{2}\left(\frac{\Gamma_{a}}{45 \mathrm{GeV}}\right)^{1 / 2} .
$$

In this case, the required values for the effective couplings of the pseudo-scalar can be weaker than the case with pseudo-scalar resonance only. When scalars decay only into 

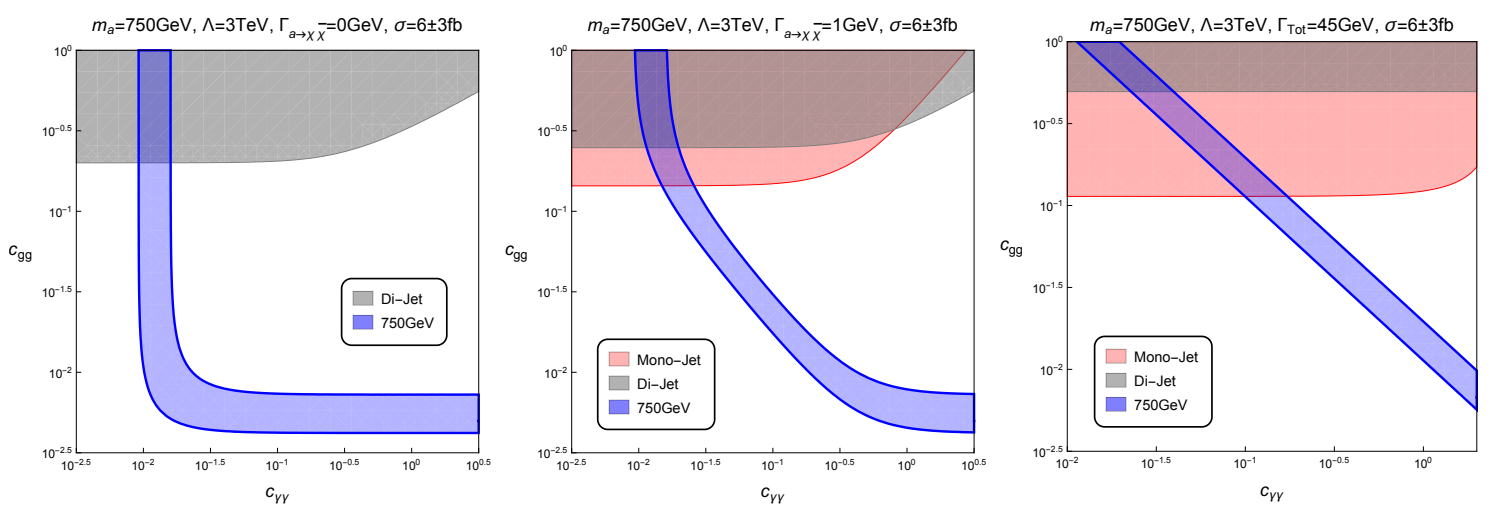

Figure 2. Parameter space of $c_{\gamma \gamma}$ and $c_{g g}$ for the diphoton resonance, from the decay of the pseudoscalar. We have taken $c_{2}=0, c_{1} \neq 0$ and $c_{3} \neq 0$ in the unbroken phase. The region explaining the diphoton resonance at $750 \mathrm{GeV}$ for $\sigma(p p \rightarrow \gamma \gamma)=6 \pm 3 \mathrm{fb}$ is shown in blue strip. The regions excluded by mono-jet and di-jet limits from LHC $8 \mathrm{TeV}$ are shown in pink (less dark) and gray (dark), respectively. The invisible decay width of the pseudo-scalar is chosen to $\Gamma_{\text {inv }}=0,1 \mathrm{GeV}$ in the left-most and middle plots, respectively, and the total decay rate is $\Gamma_{\text {tot }}=45 \mathrm{GeV}$ in the right-most plot. The case with real-scalar resonance is similar.

a pair of SM gauge bosons, the left-hand side in eq. (3.6) becomes $\sqrt{2}\left|c_{g g} \cdot c_{\gamma \gamma}\right|$, so the required effective couplings are reduced accordingly.

We remark on the important collider bounds on the model from the LHC. First, the mono-jet bound from CMS $8 \mathrm{TeV}[72]$ is given by

$$
\sigma(p p \rightarrow X \rightarrow \chi \bar{\chi})<0.8 \mathrm{pb}
$$

which is translated to the bound on the ratio of the partial decays at $\mathrm{LHC} 13 \mathrm{TeV}$,

$$
\frac{\Gamma(a \rightarrow \chi \bar{\chi})}{\Gamma(a \rightarrow \gamma \gamma)}<667\left(\frac{r}{5}\right)\left(\frac{6 \mathrm{fb}}{\sigma(p p \rightarrow \gamma \gamma)}\right)
$$

where $r$ is the parton luminosity ratio given by $r=\left(C_{g g} / s\right)_{13 \mathrm{TeV}} /\left(C_{g g} / s\right)_{8 \mathrm{TeV}} \simeq 4.7$. Then, for $r=4.7$ and $\sigma(p p \rightarrow \gamma \gamma)=6 \mathrm{fb}$, the mono-jet bound constrains the dark matter coupling to the resonance as

$$
\frac{\lambda_{\chi}^{2}}{4 c_{\gamma \gamma}^{2}} \frac{\Lambda^{2}}{m_{a}^{2}} \lesssim 627
$$

For $\Lambda=3 \mathrm{TeV}$ and $m_{a}=750 \mathrm{GeV}$, we get

$$
\left|\lambda_{\chi}\right| \lesssim 13\left|c_{\gamma \gamma}\right|
$$

For $\left|c_{\gamma \gamma}\right|=\mathcal{O}(1)$, the mono-jet bound does not constrain the dark matter coupling much, but the case is strongly limited by indirect detection such as Fermi-LAT gamma-ray searches as will be discussed in the next section.

Furthermore, the di-jet bound at LHC $8 \mathrm{TeV}, \sigma(p p \rightarrow X \rightarrow j j)<2.5 \mathrm{pb}$ [73], constrains the pseudo-scalar couplings by

$$
\frac{\Gamma(a \rightarrow g g)}{\Gamma(a \rightarrow \gamma \gamma)} \lesssim 2083\left(\frac{r}{5}\right)\left(\frac{6 \mathrm{fb}}{\sigma(p p \rightarrow \gamma \gamma)}\right)
$$




\begin{tabular}{|c||c|c|c|c|c|c|}
\hline Model & $\mathrm{BR}_{a}(\gamma \gamma)$ & $\mathrm{BR}_{a}(g g)$ & $\mathrm{BR}_{a}(Z \gamma)$ & $\mathrm{BR}_{a}(Z Z)$ & $\mathrm{BR}_{a}(\chi \bar{\chi})$ & $\Gamma_{a, \text { tot }}[\mathrm{GeV}]$ \\
\hline $\mathrm{A}$ & $5.31 \times 10^{-4}$ & 0.840 & $3.07 \times 10^{-4}$ & $4.36 \times 10^{-5}$ & 0.159 & 1.42 \\
\hline $\mathrm{B}$ & $2.12 \times 10^{-3}$ & 0.016 & $1.23 \times 10^{-3}$ & $1.74 \times 10^{-4}$ & 0.980 & 18.6 \\
\hline $\mathrm{C}$ & $2.12 \times 10^{-3}$ & 0.996 & $1.23 \times 10^{-3}$ & $1.74 \times 10^{-4}$ & - & 0.30 \\
\hline
\end{tabular}

Table 1. Decay branching fractions and total decay rate of pseudo-scalar resonance. Benchmark models with $\left(c_{g g}, m_{\chi}, \lambda_{\chi}\right)$ are Model A: $(0.2,347 \mathrm{GeV}, 0.2)$; Model B: $(0.1,293 \mathrm{GeV}, 1.4)$; Model $\mathrm{C}:(0.1,800 \mathrm{GeV}, 1.8)$. The diphoton condition (3.3) with $\sigma(p p \rightarrow \gamma \gamma)=6 \mathrm{fb}$ leads to $c_{\gamma \gamma}=$ $0.0142,0.103,0.0130$, in the order of models. We have taken $c_{2}=0, c_{1} \neq 0$ and $c_{3} \neq 0$ in the unbroken phase. For all models, we have taken $m_{a}=750 \mathrm{GeV}$ and the current collider bounds are fullfilled. These benchmark models will be used for dark matter discussion in table 4 in section 4 .

\begin{tabular}{|c||c|c|c|c|c|c|}
\hline Model & $\mathrm{BR}_{s}(\gamma \gamma)$ & $\mathrm{BR}_{s}(g g)$ & $\mathrm{BR}_{s}(Z \gamma)$ & $\mathrm{BR}_{s}(Z Z)$ & $\mathrm{BR}_{s}(\chi \bar{\chi})$ & $\Gamma_{s, \text { tot }}[\mathrm{GeV}]$ \\
\hline $\mathrm{A}$ & $5.31 \times 10^{-4}$ & 0.941 & $3.07 \times 10^{-4}$ & $4.36 \times 10^{-5}$ & $5.83 \times 10^{-2}$ & 1.27 \\
\hline $\mathrm{B}$ & $5.31 \times 10^{-4}$ & 0.999 & $3.07 \times 10^{-4}$ & $4.36 \times 10^{-5}$ & - & 1.19 \\
\hline $\mathrm{C}$ & 0.0785 & $1.70 \times 10^{-4}$ & 0.0438 & $6.03 \times 10^{-3}$ & 0.872 & 47.5 \\
\hline
\end{tabular}

Table 2. Decay branching fractions and total decay rate of real-scalar resonance. Benchmark models are Model A: $\left(d_{g g}, m_{\chi}, \lambda_{\chi}\right)=(0.2,361 \mathrm{GeV}, 0.5)$; Model B: $\left(d_{g g}, m_{\chi}, \lambda_{\chi}\right)=(0.2,800 \mathrm{GeV}, 1.4)$; Model C: $\left(d_{\gamma \gamma}, m_{\chi}, \lambda_{\chi}\right)=(1.0,265 \mathrm{GeV}, 2.8)$. The diphoton condition (3.3) for $\sigma(p p \rightarrow \gamma \gamma)=6 \mathrm{fb}$ with $c_{g g}, c_{\gamma \gamma}$ being replaced by $d_{g g}, d_{\gamma \gamma}$, respectively, leads to $d_{\gamma \gamma}=0.0134,0.0130, d_{g g}=0.0165$, in the order of models. We have taken $c_{2}=0, c_{1} \neq 0$ and $c_{3} \neq 0$ in the unbroken phase. For all models, we have taken $m_{s}=750 \mathrm{GeV}$ and the current collider bounds are fulfilled. These benchmark models will be used for dark matter discussion in table 5 in section 5 .

Thus, for $r=4.7$ and $\sigma(p p \rightarrow \gamma \gamma)=6 \mathrm{fb}$, the di-jet bound constrains the gluon coupling to the resonance as

$$
\left|c_{g g}\right| \lesssim 15.6\left|c_{\gamma \gamma}\right|
$$

When the real scalar is the diphoton resonance, a similar bound on the gluon coupling $d_{g g}$ applies.

In tables 1 and 2, we show the branching fractions and total decay rates of the pseudoscalar and real-scalar resonances, respectively, in some benchmark models with dark matter couplings, satisfying the diphoton condition as well as the above current collider bounds.

\subsection{Diphotons from cascade decay}

The ATLAS ECAL is located at $r=1.5$ meters from the beam and the CMS ECAL lies at $r=1.3$ meters. The cell size of ECAL detectors in CMS and ATLAS is about $\eta=0.0174$ and 0.025 in pseudo-rapidity, respectively. We also note that the first layer of the ATLAS ECAL ranges between 0.003 and 0.006 depending on $\eta$. So, if $|\Delta \eta|$ between two photons is smaller than the ECAL cell size, two photons would hit the same ECAL cell so they are identified as a single photon in the ECAL detector [50-57].

Suppose that diphotons come from the cascade decay of the resonance through light intermediate particles [50-57], namely, $X \rightarrow Y Y \rightarrow 4 \gamma$ with $Y \rightarrow \gamma \gamma$. Then, for $m_{X} \gg m_{Y}$, 
the decay length of the $Y$ particle is given by

$$
d=\left(c \tau_{Y}\right) \gamma \approx \frac{1}{\Gamma_{Y}} \frac{E_{Y}}{m_{Y}}
$$

with $\gamma=E_{Y} / m_{Y}$. On the other hand, the pseudo-rapidity separation between a photon pair coming from the decay of the $Y$ particle is given by

$$
|\Delta \eta| \approx \frac{2 m_{Y}}{E_{Y}}\left(1-\frac{d}{r}\right) .
$$

For instance, for $d \lesssim r$ and $|\Delta \eta|<0.003$, taking $E_{Y}=m_{X} / 2$ with $m_{X}=750 \mathrm{GeV}$, we need $m_{Y} \lesssim 0.5 \mathrm{GeV}$. In this case, two photons coming from the decay of each $Y$ are collimated and are considered as a singlet photon in the detector. Then, the resonance production cross section of particle $X$ with cascade decays is

$$
\sigma(p p \rightarrow X \rightarrow 4 \gamma)=\frac{1}{s M_{X} \Gamma_{X}} K_{g g} C_{g g} \Gamma(X \rightarrow g g) \Gamma(X \rightarrow Y Y)(\mathrm{BR}(Y \rightarrow \gamma \gamma))^{2} .
$$

We note that the $Y$ couplings to gauge bosons can be small enough as far as the decay length is smaller than the ECAL radius. Thus, the bounds on a light scalar at the LEP or LHC can be evaded.

In our model, we choose a real scalar resonance of $750 \mathrm{GeV}$ and a light pseudo-scalar having mass $m_{a}$, namely, $X=h_{2}$ and $Y=a$. In figure 3 , we depict the separation of two photons $|\Delta \eta|$ and the decay length $d$ for the decay of the pseudo-scalar in the parameter space for the mass and photon coupling of the pseudo-scalar. For a lot of the parameter space, the pseudo-scalar can decay well within the ECAL radius. For instance, for $m_{2}=750 \mathrm{GeV}$ and $m_{a}=0.4 \mathrm{GeV}$, we need the photon coupling to be $c_{\gamma \gamma}>0.028$ for $d<1.5 \mathrm{~m}$. Moreover, the region of the parameter space with $c_{\gamma \gamma} \lesssim 0.4$ shown for the cascade decay in figure 3 is consistent with the previous limits from $e^{+} e^{-} \rightarrow \gamma^{*} / Z^{(*)} \rightarrow a \gamma$ with $a \rightarrow 2 \gamma$ in LEP [74], in particular, at the $Z$-peak. ${ }^{2}$ But, LHC and future colliders such as FCC-ee would be able to probe the photon coupling $c_{\gamma \gamma}$ of order 0.01 for sub-GeV masses from the same process [74].

With the contribution from the direct decay of the resonance into two photons included in our model, the observed diphoton production cross section leads to

$$
\Gamma_{2}(g g)\left[\Gamma_{2}(\gamma \gamma)+\Gamma_{2}(a a)(\operatorname{BR}(a \rightarrow \gamma \gamma))^{2}\right]=2.8 \times 10^{-2} \mathrm{GeV}^{2}\left(\frac{\sigma(p p \rightarrow \gamma \gamma)}{6 \mathrm{fb}}\right)\left(\frac{\Gamma_{2}}{45 \mathrm{GeV}}\right)
$$

In this case, a small gluon coupling is allowed for a sizable partial decay rate of the realscalar into a pair of pseudo-scalars, as far as $\operatorname{BR}(a \rightarrow \gamma \gamma)$ is sizable. In figure 4 , we show the parameter space for $c_{g g}$ vs $c_{\gamma \gamma}$ by including the cascade decay contribution to the diphoton excess, denoted by the ratio of cascade to direct decay into photons, $R \equiv \Gamma_{2}(a a)(\operatorname{BR}(a \rightarrow$ $\gamma \gamma))^{2} / \Gamma_{2}(\gamma \gamma)$. For $\operatorname{BR}(a \rightarrow \gamma \gamma)=1$, we have taken ${ }^{3} m_{a} \lesssim 3 m_{\pi}$, namely, $m_{a}=0.4 \mathrm{GeV}$.

\footnotetext{
${ }^{2}$ The hypercharge coupling to the pseudo-scalar can lead to the $Z \gamma$ coupling as well as the $\gamma \gamma$ coupling.

${ }^{3}$ For $m_{a}>3 m_{\pi}$, the pseudo-scalar decays into three pions or mesons, so $\operatorname{BR}(a \rightarrow \gamma \gamma)$ gets suppressed. In this case, the cascade contribution to diphoton excesses is sub-dominant. Furthermore, the $\eta$ separation between collimated photons becomes larger than 0.003 for $m_{a} \gtrsim 0.5 \mathrm{GeV}$.
} 


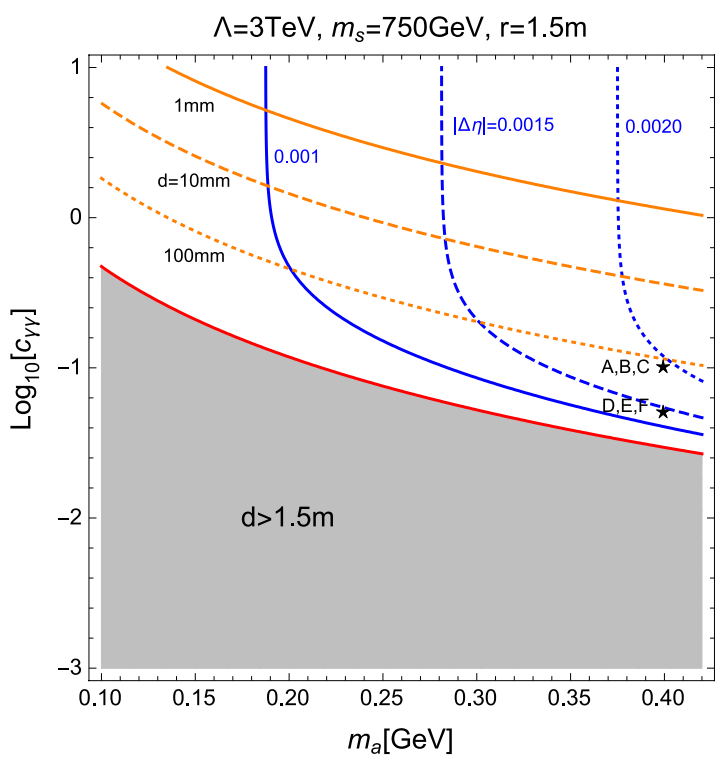

Figure 3. Contours for separation of two photons in pseudo-rapidity $|\Delta \eta|$ and decay length $d$ in the parameter space of the mass $m_{a}$ and photon coupling $c_{\gamma \gamma}$ of the light pseudo-scalar. $\Lambda=3 \mathrm{TeV}$ and $m_{s}=750 \mathrm{GeV}$ are taken, while $c_{2}=0, c_{1} \neq 0$ and $c_{3} \neq 0$ is taken in the unbroken phase. The radius of ECAL is chosen to $r=1.5 \mathrm{~m}$. The region with $d>r=1.5 \mathrm{~m}$ is shown in gray, with boundary $d=1.5 \mathrm{~m}$ in red line. We have shown benchmark models in star, as will be discussed in table 3 .

Keeping the total width of the real-scalar resonance to $\Gamma_{s, \text { tot }}=45 \mathrm{GeV}$, we vary the singlet quartic coupling $\lambda_{S}=0.01,0.1,1$, from left to right figures in figure 4 , and show that the parameter space with cascade decay dominance increases, being compatible with mono-jet and di-jet bounds. In the case with a sizable cascade decay, the gluon coupling is more or less fixed to a small value while there is a little dependence on the photon coupling as far as the photon-jet contribution is dominant.

When the diphoton resonance is dominated by the cascade decay, the condition on the effective couplings of the resonance becomes

$$
\left|d_{g g}\right| \sqrt{\lambda_{S}}=0.071\left(\frac{\sigma(p p \rightarrow \gamma \gamma)}{6 \mathrm{fb}}\right)^{1 / 2}\left(\frac{\Lambda}{3 \mathrm{TeV}}\right)^{2}\left(\frac{\Gamma_{2}}{45 \mathrm{GeV}}\right)^{1 / 2}\left(\frac{1 / 9}{\operatorname{BR}(a \rightarrow \gamma \gamma)}\right)
$$

where use is made of $m_{2}=\sqrt{2 \lambda_{S}} v_{s}$ and $m_{a} \ll m_{s} / 2$. In the decoupled vector-like fermions with the same global charges as dark matter, we get $d_{g g}=\frac{4}{3} c_{g g}$, so the above condition becomes

$$
\left|c_{g g}\right| \sqrt{\lambda_{S}}=0.053\left(\frac{\sigma(p p \rightarrow \gamma \gamma)}{6 \mathrm{fb}}\right)^{1 / 2}\left(\frac{\Lambda}{3 \mathrm{TeV}}\right)^{2}\left(\frac{\Gamma_{2}}{45 \mathrm{GeV}}\right)^{1 / 2}\left(\frac{1 / 9}{\operatorname{BR}(a \rightarrow \gamma \gamma)}\right)
$$

The mono-jet bound from CMS $8 \mathrm{TeV}$ for the case with cascade decay is given as follows,

$$
\frac{\Gamma_{2}(\chi \bar{\chi})}{\Gamma_{2}(\gamma \gamma)+\Gamma_{2}(a a)(\operatorname{BR}(a \rightarrow \gamma \gamma))^{2}}<667\left(\frac{r}{5}\right)\left(\frac{6 \mathrm{fb}}{\sigma(p p \rightarrow \gamma \gamma)}\right)
$$



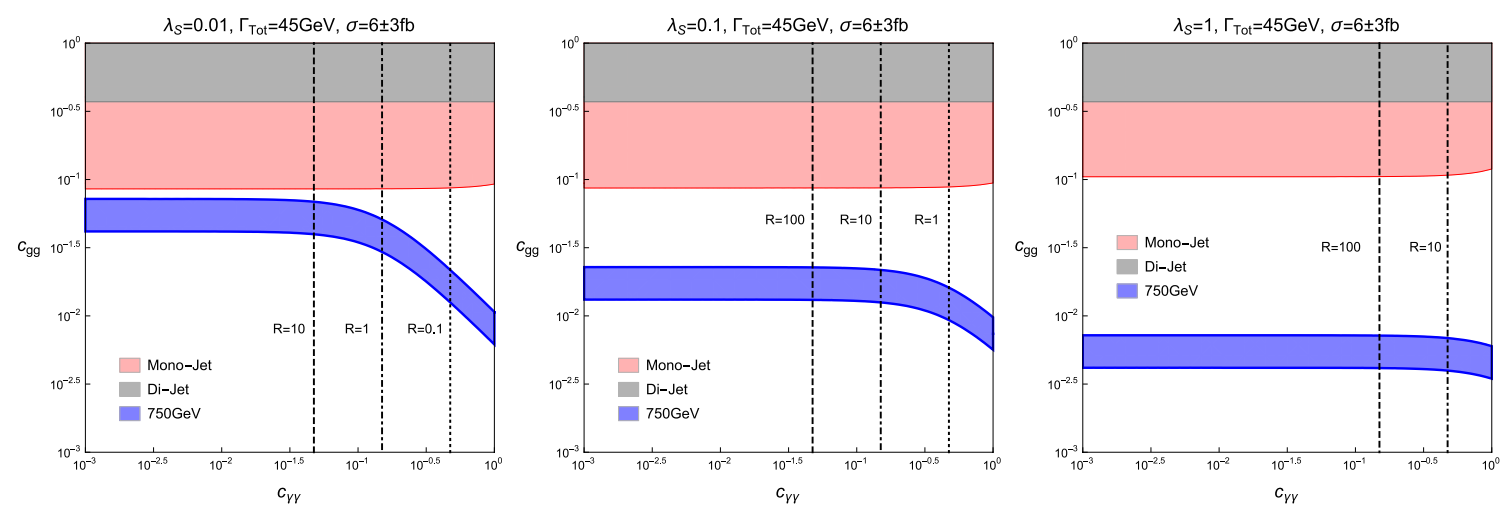

Figure 4. Parameter space of $c_{\gamma \gamma}$ and $c_{g g}$ for the diphoton resonance, including both direct and cascade decays of the real scalar. We have taken $c_{2}=0, c_{1} \neq 0$ and $c_{3} \neq 0$ in the unbroken phase. We have chosen $m_{s}=750 \mathrm{GeV}$ and $m_{a}=0.4 \mathrm{GeV}$. The region explaining the diphoton resonance at $750 \mathrm{GeV}$ for $\sigma(p p \rightarrow \gamma \gamma)=6 \pm 3 \mathrm{fb}$ is shown in blue. Several values of the ratio of cascade to direct decay rates, $R$, are shown in each plot. The regions excluded by mono-jet and di-jet limits from LHC $8 \mathrm{TeV}$ are shown in pink and gray, respectively. The quartic coupling of the real scalar is chosen to $\lambda_{S}=0.01,0.1,1.0$, from left to right plots. In all the plots, the total decay rate of the real scalar, including the invisible decay mode, is fixed to $\Gamma_{\text {tot }}=45 \mathrm{GeV}$.

When the cascade decay is dominant, for $r=4.7$ and $\sigma(p p \rightarrow \gamma \gamma)=6 \mathrm{fb}$, we get the mono-jet bound on the dark matter coupling to the resonance as

$$
\frac{\lambda_{\chi}^{2} m_{2}^{2}}{2 \lambda_{S}^{2} v_{s}^{2}} \lesssim 9.80\left(\frac{\mathrm{BR}(a \rightarrow \gamma \gamma)}{1 / 9}\right)^{2}
$$

which becomes, for $\Lambda=3 \mathrm{TeV}$ and $m_{2}=750 \mathrm{GeV}$,

$$
\left|\lambda_{\chi}\right| \lesssim 3.13 \sqrt{\lambda_{S}}\left(\frac{\operatorname{BR}(a \rightarrow \gamma \gamma)}{1 / 9}\right)
$$

Here, use is made of $m_{2}=\sqrt{2 \lambda_{S}} v_{s}$ in the limit of a vanishing Higgs mixing angle.

On the other hand, the di-jet bound at LHC $8 \mathrm{TeV}$ leads to

$$
\frac{\Gamma_{2}(g g)+\Gamma_{2}(a a)(\operatorname{BR}(a \rightarrow g g))^{2}}{\Gamma_{2}(\gamma \gamma)+\Gamma_{2}(a a)(\operatorname{BR}(a \rightarrow \gamma \gamma))^{2}} \lesssim 2083\left(\frac{r}{5}\right)\left(\frac{6 \mathrm{fb}}{\sigma(p p \rightarrow \gamma \gamma)}\right)
$$

When the cascade decay is dominant, for $r=4.7$ and $\sigma(p p \rightarrow \gamma \gamma)=6 \mathrm{fb}$, the gluon coupling to the real scalar resonance is constrained by

$$
\left|c_{g g}\right| \lesssim 2.35\left|c_{\gamma \gamma}\right|
$$

Therefore, the gluon coupling is much more constrained, as compared to the case with direct decay where $\left|c_{g g}\right| \lesssim 15.6\left|c_{\gamma \gamma}\right|$ is obtained from the di-jet bound.

On the other hand, if the pseudo-scalar is lighter than $3 m_{\pi} \sim 420 \mathrm{MeV}$ we get $\operatorname{BR}(a \rightarrow$ $\gamma \gamma)=1$. In this case, there is no extra di-jet from the cascade decays. Then, for $r=4.7$, $\sigma(p p \rightarrow \gamma \gamma)=6 \mathrm{fb}, \Lambda=3 \mathrm{TeV}$ and $m_{2}=750 \mathrm{GeV}$, the dijet bound leads to $\left|d_{g g}\right| \lesssim 32 \sqrt{\lambda_{S}}$. 


\begin{tabular}{|c||c|c|c|c|}
\hline Model & $\mathrm{BR}_{s}(\gamma \gamma)$ & $\mathrm{BR}_{s}(g g)$ & $\mathrm{BR}_{s}(Z \gamma)$ & $\mathrm{BR}_{s}(Z Z)$ \\
\hline $\mathrm{A}$ & $6.59 \times 10^{-3}$ & $3.99 \times 10^{-4}$ & $3.81 \times 10^{-3}$ & $5.42 \times 10^{-4}$ \\
\hline $\mathrm{B}$ & $4.14 \times 10^{-2}$ & $3.99 \times 10^{-4}$ & $2.39 \times 10^{-2}$ & $3.40 \times 10^{-3}$ \\
\hline $\mathrm{C}$ & $1.68 \times 10^{-3}$ & $4.15 \times 10^{-5}$ & $9.71 \times 10^{-4}$ & $1.38 \times 10^{-4}$ \\
\hline $\mathrm{D}$ & $1.99 \times 10^{-3}$ & $4.12 \times 10^{-4}$ & $1.15 \times 10^{-3}$ & $1.64 \times 10^{-4}$ \\
\hline $\mathrm{E}$ & $1.09 \times 10^{-2}$ & $4.12 \times 10^{-4}$ & $6.31 \times 10^{-3}$ & $8.97 \times 10^{-4}$ \\
\hline $\mathrm{F}$ & $4.38 \times 10^{-4}$ & $4.17 \times 10^{-5}$ & $2.53 \times 10^{-4}$ & $3.60 \times 10^{-5}$ \\
\hline
\end{tabular}

\begin{tabular}{|c||c|c|c|c|}
\hline Model & $\mathrm{BR}_{s}(\chi \bar{\chi})$ & $\mathrm{BR}_{s}(a a)$ & $\Gamma_{s, \text { tot }}$ & $\Gamma_{a, \text { tot }}$ \\
\hline $\mathrm{A}$ & 0.840 & 0.148 & 10.1 & $5.66 \times 10^{-12}$ \\
\hline $\mathrm{B}$ & - & 0.931 & 1.60 & $5.66 \times 10^{-12}$ \\
\hline $\mathrm{C}$ & 0.620 & 0.378 & 39.5 & $5.66 \times 10^{-12}$ \\
\hline $\mathrm{D}$ & 0.817 & 0.167 & 8.32 & $1.42 \times 10^{-12}$ \\
\hline $\mathrm{E}$ & - & 0.982 & 1.52 & $1.42 \times 10^{-12}$ \\
\hline $\mathrm{F}$ & 0.605 & 0.394 & 37.9 & $1.42 \times 10^{-12}$ \\
\hline
\end{tabular}

Table 3. Decay branching fractions and total decay rates (in units of $\mathrm{GeV}$ ) of realscalar and pseudo-scalar, when the former is the $750 \mathrm{GeV}$ resonance. Benchmark models with $\left(c_{\gamma \gamma}, m_{\chi}, \lambda_{\chi}, \lambda_{s}, c_{g g}\right)$ are Model A: $\left(0.1,320 \mathrm{GeV}, 2.0,0.1,4.29 \times 10^{-3}\right)$, Model B: $\left(0.1,950 \mathrm{GeV}, 1.0,0.1,3.47 \times 10^{-3}\right)$, Model C: $\left(0.1,190 \mathrm{GeV}, 1.6,1.0,5.56 \times 10^{-3}\right)$, Model D: $\left(0.05,328 \mathrm{GeV}, 2.0,0.1,3.44 \times 10^{-3}\right)$, Model E: $\left(0.05,920 \mathrm{GeV}, 1.0,0.1,3.44 \times 10^{-3}\right)$, Model F: $\left(0.05,220 \mathrm{GeV}, 1.7,1.0,3.43 \times 10^{-3}\right)$. We have taken $c_{2}=0, c_{1} \neq 0$ and $c_{3} \neq 0$ in the unbroken phase. For all models, we have imposed $\sigma(p p \rightarrow \gamma \gamma)=6 \mathrm{fb}$ for $m_{s}=750 \mathrm{GeV}$ and $m_{a}=0.4 \mathrm{GeV}$ while the current collider bounds and $d<1.5 \mathrm{~m}$ are fulfilled. These benchmark models will be used for dark matter discussion in table 6 in section 6 .

In the presence of cascade decay of the real scalar, in table 3, we show the branching fractions and total decay rate of the pseudo-scalar and real-scalar, respectively, in some benchmark models with dark matter couplings, that satisfy the diphoton condition as well as the above current collider bounds. Here, we have set the scalar masses to $m_{s}=750 \mathrm{GeV}$ and $m_{a}=0.4 \mathrm{GeV}$ below the pion threshold such that $\operatorname{BR}(a \rightarrow \gamma \gamma)=1$, and the effective gauge couplings are taken to $d_{i}=\frac{4}{3} c_{i}(i=1,3)$ and $c_{2}=d_{2}=0$. The photon couplings in all the models are within the reach of the future colliders.

\section{Dark matter with pseudo-scalar resonance}

In this section, we interpret the diphoton resonance by the direct decay of the pseudoscalar in our model, focusing on the pseudo-scalar coupling to a Dirac singlet fermion dark matter. The DM relic density condition, the constraints from indirect detection for dark matter and the mono-jet limits are superimposed.

\subsection{Dark matter annihilation}

When the real scalar is heavy, we can consider the interactions of the pseudo-scalar field only in the Lagrangian (2.2). Then, the pseudo-scalar can play a role of mediator between dark matter and the SM [58-61]. When vector-like fermions are sufficiently heavier than 


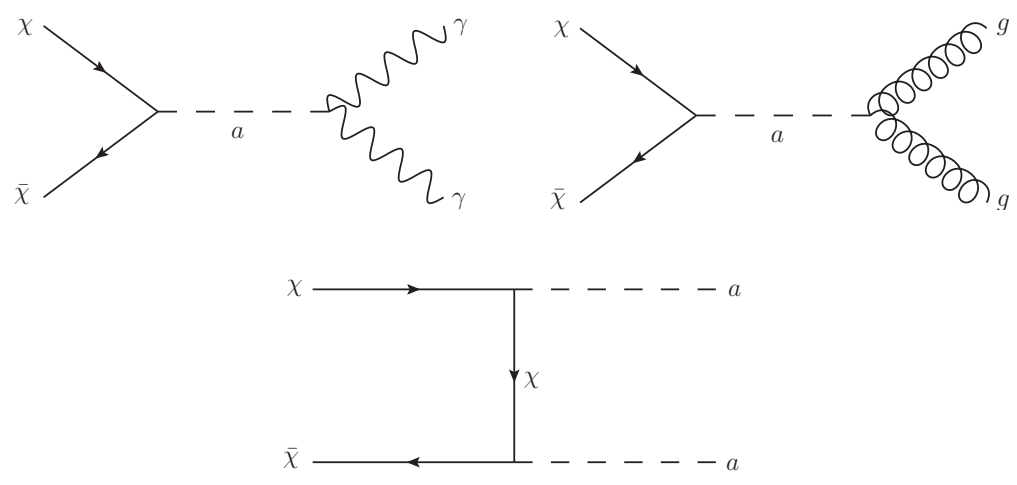

Figure 5. Feynman diagrams for dark matter annihilation with pseudo-scalar resonance.

dark matter, we can use the effective interactions for pseudo-scalar resonance in the process of dark matter annihilation as shown in figure 5. In this case, the total annihilation cross section of dark matter is given by $\left(\sigma v_{\text {rel }}\right)_{a}=\sum_{i}\left(\sigma v_{\text {rel }}\right)_{a, i}+\left(\sigma v_{\text {rel }}\right)_{a a}$ with partial annihilation cross sections into a pair of SM gauge bosons being

$$
\begin{aligned}
\left(\sigma v_{\mathrm{rel}}\right)_{a, g g} & =\frac{16 \lambda_{\chi}^{2} c_{g g}^{2}}{\pi \Lambda^{2}} \frac{m_{\chi}^{4}}{\left(4 m_{\chi}^{2}-m_{a}^{2}\right)^{2}+\Gamma_{a}^{2} m_{a}^{2}} \\
\left(\sigma v_{\mathrm{rel}}\right)_{a, \gamma \gamma} & =\frac{2 \lambda_{\chi}^{2} c_{\gamma \gamma}^{2}}{\pi \Lambda^{2}} \frac{m_{\chi}^{4}}{\left(4 m_{\chi}^{2}-m_{a}^{2}\right)^{2}+\Gamma_{a}^{2} m_{a}^{2}} \\
\left(\sigma v_{\mathrm{rel}}\right)_{a, Z \gamma} & =\frac{\lambda_{\chi}^{2} c_{Z \gamma}^{2}}{\pi \Lambda^{2}} \frac{m_{\chi}^{4}}{\left(4 m_{\chi}^{2}-m_{a}^{2}\right)^{2}+\Gamma_{a}^{2} m_{a}^{2}}\left(1-\frac{m_{Z}^{2}}{4 m_{\chi}^{2}}\right)^{3} \\
\left(\sigma v_{\mathrm{rel}}\right)_{a, Z Z} & =\frac{2 \lambda_{\chi}^{2} c_{Z Z}^{2}}{\pi \Lambda^{2}} \frac{m_{\chi}^{4}}{\left(4 m_{\chi}^{2}-m_{a}^{2}\right)^{2}+\Gamma_{a}^{2} m_{a}^{2}}\left(1-\frac{m_{Z}^{2}}{m_{\chi}^{2}}\right)^{3 / 2} \\
\left(\sigma v_{\mathrm{rel}}\right)_{a, W W} & =\frac{\lambda_{\chi}^{2} c_{W W}^{2}}{\pi \Lambda^{2}} \frac{m_{\chi}^{4}}{\left(4 m_{\chi}^{2}-m_{a}^{2}\right)^{2}+\Gamma_{a}^{2} m_{a}^{2}}\left(1-\frac{m_{W}^{2}}{m_{\chi}^{2}}\right)^{3 / 2}
\end{aligned}
$$

where

$$
\begin{aligned}
c_{g g} & =c_{3}, \\
c_{\gamma \gamma} & =c_{1} \cos ^{2} \theta_{W}+c_{2} \sin ^{2} \theta_{W}, \\
c_{Z \gamma} & =\left(c_{2}-c_{1}\right) \sin \left(2 \theta_{W}\right), \\
c_{Z Z} & =c_{1} \sin ^{2} \theta_{W}+c_{2} \cos ^{2} \theta_{W}, \\
c_{W W} & =2 c_{2} .
\end{aligned}
$$

We note that all the gauge boson channels are s-wave.

For $m_{\chi}>m_{a}$, dark matter can annihilate into a pair of pseudo-scalars. In the limit of non-relativistic dark matter, the corresponding annihilation cross sections for the $a a$ 
channel becomes

$$
\begin{aligned}
\left(\sigma v_{\text {rel }}\right)_{a a}= & \frac{\lambda_{\chi}^{2}}{96 \pi}\left[\frac{\lambda_{\chi}^{2} m_{\chi}^{6}}{\left(m_{a}^{2}-2 m_{\chi}^{2}\right)^{4}}\left(1-\frac{m_{a}^{2}}{m_{\chi}^{2}}\right)^{2}+\frac{3 \lambda_{S}^{2} v_{s}^{2}}{2\left[\left(4 m_{\chi}^{2}-m_{s}^{2}\right)^{2}+\Gamma_{s}^{2} m_{s}^{2}\right]}\right. \\
& \left.+\frac{\sqrt{2} \lambda_{\chi} \lambda_{S} v_{s} m_{\chi}^{3}}{\left(m_{a}^{2}-2 m_{\chi}^{2}\right)^{2}} \cdot \frac{4 m_{\chi}^{2}-m_{s}^{2}}{\left(4 m_{\chi}^{2}-m_{s}^{2}\right)^{2}+\Gamma_{s}^{2} m_{s}^{2}}\left(1-\frac{m_{a}^{2}}{m_{\chi}^{2}}\right)\right]\left(1-\frac{m_{a}^{2}}{m_{\chi}^{2}}\right)^{1 / 2} v_{\text {rel }}^{2}
\end{aligned}
$$

Here, we have also included the real-scalar contribution to the $a a$ channel, for a later use with real and pseudo-scalars in the effective field theory in section 6 . Thus, the $a a$ channel turns out to be $p$-wave suppressed, so they are not relevant for indirect detection at present. However, the $a a$ channel, if open kinematically, still contributes to the thermal cross section at freeze-out.

\subsection{Bounds from indirect detections}

The cosmic ray flux stemming from the annihilation of a Dirac fermion dark matter into $f$ final states (such as $\gamma$-ray, $e^{+}, p^{+}, \nu$, etc) is given by

$$
\frac{d \Phi_{f}}{d E_{f}}=\frac{1}{16 \pi m_{\chi}^{2}}\langle\sigma v\rangle_{f} \frac{d N_{f}}{d E_{f}} J
$$

where $\frac{d N_{f}}{d E}$ is the differential cosmic-ray yield per annihilation and the J-factor is the lineof-sight integral through the dark matter distribution integrated over the solid angle $\Delta \Omega$, given by

$$
J=\frac{1}{\Delta \Omega} \int_{\Delta \Omega} d \Omega \int_{\text {l.o.s. }} d s \rho_{\chi}^{2}
$$

On the other hand, the loop-induced interactions of the pseudo-scalar to gluons lead to the effective interactions between dark matter and gluon fields. Thus, gluons inside nucleons can scatter off with dark matter, leading to recoil energy signals in underground experiments. But, the current dark matter experiments are not sensitive enough to detect the signals. Therefore, henceforth we focus on the indirect detection.

Dark matter annihilation channels, $\chi \bar{\chi} \rightarrow a \rightarrow \gamma \gamma, Z \gamma$, are $s$-wave and they lead to monochromatic photons at $E_{\gamma}=m_{\chi}$ and $E_{\gamma}=m_{\chi}\left(1-\frac{4 m_{Z}^{2}}{m_{\chi}^{2}}\right)$, respectively. Those channels can be constrained by Fermi-LAT [75-78] and HESS [82] line searches from the galactic center.

Annihilation channels of dark matter into $W W, Z Z, g g$ lead to continuum photons from bremstrahlung or decay and they are constrained by Fermi-diffuse gamma-ray searches from dwarf galaxies [79-81]. Moreover, dark matter annihilation into a pair of gluons can be constrained by anti-proton data from PAMELA and AMS-02 [83-86].

In figure 6 , we show the parameter space of dark matter mass $m_{\chi}$ and coupling $\lambda_{\chi}$ in the model with pseudo-scalar resonance where the condition for diphoton excesses is satisfied. Depending on the value of the gluon coupling $c_{g g}=0.2(0.1)$ on left (right) plots, respectively, with the photon coupling $c_{\gamma \gamma}$ being determined by the diphoton condition (3.3), we imposed the current bounds from mono-jet searches as well as the indirect detections. 

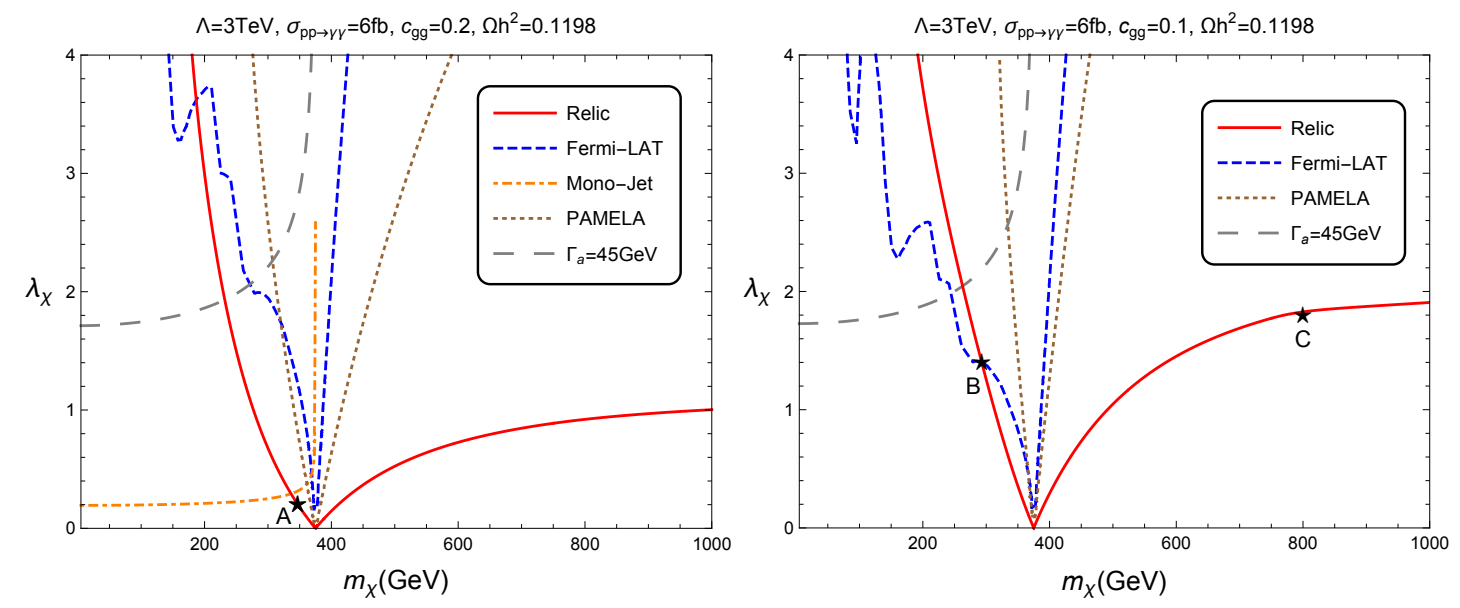

Figure 6. Parameter space of $m_{\chi}$ and $\lambda_{\chi}$ with pseudo-scalar mediator, satisfying the relic density in red lines. The region explaining the diphoton resonance at $750 \mathrm{GeV}$ for $\sigma(p p \rightarrow \gamma \gamma)=6 \mathrm{fb}$ is imposed. $c_{g g}=0.2$ and $c_{g g}=0.1$ are chosen on left and right, while $c_{2}=0$ in both plots. The mono-jet limit from LHC $8 \mathrm{TeV}$ are shown in orange dot-dashed line. The region above blue dashed line and brown dotted line are excluded by the bound from Fermi-LAT line search (R16 with Einasto profile) and the antiproton bound from PAMELA. The line for $\Gamma_{a}=45 \mathrm{GeV}$ is also shown in dashed gray. Benchmark models A (B and C) are shown in star on the left (right) plot, taken in table 1.

In the former case with $c_{g g}=0.2$, the mono-jet bound is quite constraining below resonance, so only the region with small dark matter coupling near resonance survives, while the antiproton bound from PAMELA reaches closely to the region saturating the relic density and the bound from other cosmic data such as Fermi-LAT are not strong. In the latter case with $c_{g g}=0.1$, there is no mono-jet bound, but the bound from FermiLAT line search constrain most strongly the region with small dark matter masses below resonance, allowing only the small region near resonance. Therefore, the mono-jet and Fermi-LAT line searches are complementary to constraining the light dark matter. On the other hand, the region above resonance is not constrained in the region where the relic density is saturated.

In table 4, we show the averaged annihilation cross sections at present (except the one for the $a a$ channel, which is given at freeze-out) and the relic density for dark matter with pseudo-scalar mediator in some benchmark models considered in table 1, satisfying the diphoton condition as well as the above current collider bounds. Model A (B and C) belongs to the left (right) plot in figure 6 . These models satisfy the current bounds from various indirect detection experiments discussed above.

\section{Dark matter with real-scalar resonance}

In this section, we consider the real-scalar resonance for the diphoton excess and discuss the interplay with indirect detection of dark matter and mono-jet searches, similarly to the case with pseudo-scalar case. 


\begin{tabular}{|c||c|c|c|c|c|c|}
\hline Model & $\left\langle\sigma v_{\text {rel }}\right\rangle_{a, \gamma \gamma}$ & $\left\langle\sigma v_{\text {rel }}\right\rangle_{a, g g}$ & $\left\langle\sigma v_{\text {rel }}\right\rangle_{a, Z \gamma}$ & $\left\langle\sigma v_{\text {rel }}\right\rangle_{a, Z Z}$ & $\left\langle\sigma v_{\text {rel }}\right\rangle_{a a}$ & $\Omega_{\chi} h^{2}$ \\
\hline A & $1.48 \times 10^{-29}$ & $2.34 \times 10^{-26}$ & $8.50 \times 10^{-30}$ & $1.20 \times 10^{-30}$ & - & 0.122 \\
\hline B & $2.62 \times 10^{-27}$ & $1.98 \times 10^{-26}$ & $1.47 \times 10^{-27}$ & $2.02 \times 10^{-28}$ & - & 0.120 \\
\hline $\mathrm{C}$ & $4.68 \times 10^{-29}$ & $2.20 \times 10^{-26}$ & $2.80 \times 10^{-29}$ & $4.13 \times 10^{-30}$ & $6.15 \times 10^{-28}$ & 0.124 \\
\hline
\end{tabular}

Table 4. Averaged annihilation cross sections (in units of $\mathrm{cm}^{3} / \mathrm{s}$ ) at present and relic density for dark matter with pseudo-scalar, except that that the one for the $a a$ channel is given at freeze-out. The benchmark models are the same as in table 1 and figure 6 . All the constraints from the current collider and cosmic data are satisfied.
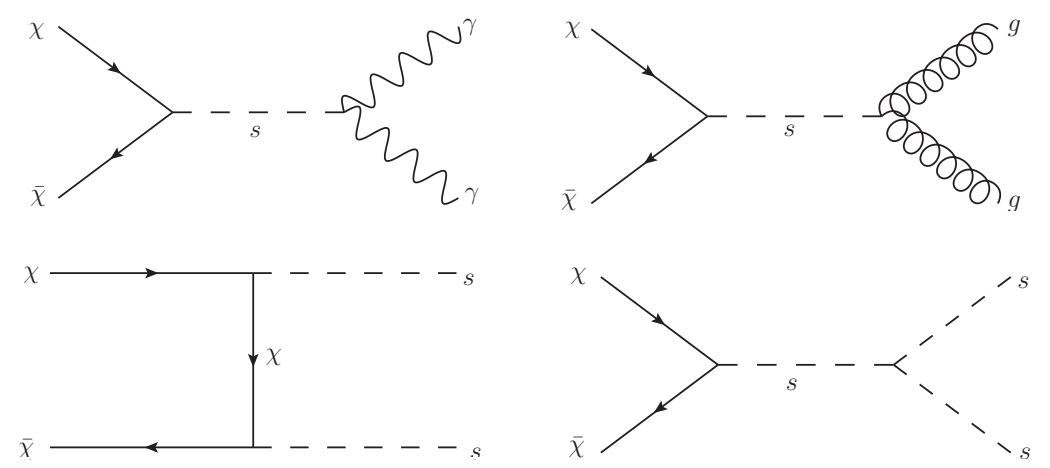

Figure 7. Feynman diagrams for dark matter annihilation with real-scalar resonance.

When the real scalar is light enough, it can contribute to the DM annihilation through s-channels and/or t-channels as shown in figure 7. Taking vector-like fermions in loops to be sufficiently heavier than dark matter, we obtain the total annihilation cross section of dark matter in terms of effective interactions for real-scalar resonance by $\left(\sigma v_{\text {rel }}\right)_{s}=$ $\sum_{i}\left(\sigma v_{\text {rel }}\right)_{s, i}+\left(\sigma v_{\text {rel }}\right)_{s s}$ with partial annihilation cross sections into a pair of SM gauge bosons being given by

$$
\begin{aligned}
\left(\sigma v_{\mathrm{rel}}\right)_{s, g g} & =\frac{4 \lambda_{\chi}^{2} d_{g g}^{2}}{\pi \Lambda^{2}} \frac{m_{\chi}^{4}}{\left(4 m_{\chi}^{2}-m_{2}^{2}\right)^{2}+\Gamma_{2}^{2} m_{2}^{2}} v_{\mathrm{rel}}^{2}, \\
\left(\sigma v_{\mathrm{rel}}\right)_{s, \gamma \gamma} & =\frac{\lambda_{\chi}^{2} d_{\gamma \gamma}^{2}}{2 \pi \Lambda^{2}} \frac{m_{\chi}^{4}}{\left(4 m_{\chi}^{2}-m_{2}^{2}\right)^{2}+\Gamma_{2}^{2} m_{2}^{2}} v_{\mathrm{rel}}^{2}, \\
\left(\sigma v_{\mathrm{rel}}\right)_{s, Z \gamma} & =\frac{\lambda_{\chi}^{2} d_{Z \gamma}^{2}}{4 \pi \Lambda^{2}} \frac{m_{\chi}^{4}}{\left(4 m_{\chi}^{2}-m_{2}^{2}\right)^{2}+\Gamma_{2}^{2} m_{2}^{2}}\left(1-\frac{m_{Z}^{2}}{4 m_{\chi}^{2}}\right)^{3} v_{\mathrm{rel}}^{2}, \\
\left(\sigma v_{\mathrm{rel}}\right)_{s, Z Z} & =\frac{\lambda_{\chi}^{2} d_{Z Z}^{2}}{2 \pi \Lambda^{2}} \frac{m_{\chi}^{4} v_{\mathrm{rel}}^{2}}{\left(4 m_{\chi}^{2}-m_{2}^{2}\right)^{2}+\Gamma_{2}^{2} m_{2}^{2}}\left(1-\frac{m_{Z}^{2}}{m_{\chi}^{2}}+\frac{3 m_{Z}^{4}}{8 m_{\chi}^{4}}\right)\left(1-\frac{m_{Z}^{2}}{m_{\chi}^{2}}\right)^{1 / 2}, \\
\left(\sigma v_{\mathrm{rel}}\right)_{s, W W} & =\frac{\lambda_{\chi}^{2} d_{W W}^{2}}{4 \pi \Lambda^{2}} \frac{m_{\chi}^{4} v_{\mathrm{rel}}^{2}}{\left(4 m_{\chi}^{2}-m_{2}^{2}\right)^{2}+\Gamma_{2}^{2} m_{2}^{2}}\left(1-\frac{m_{W}^{2}}{m_{\chi}^{2}}+\frac{3 m_{W}^{4}}{8 m_{\chi}^{4}}\right)\left(1-\frac{m_{W}^{2}}{m_{\chi}^{2}}\right)^{1 / 2} .
\end{aligned}
$$

We note that all the gauge boson channels are $p$-wave suppressed.

For $m_{\chi}>m_{s}$, dark matter can annihilate into a pair of real scalars. In the limit of non-relativistic dark matter, the corresponding annihilation cross section for the ss channel 

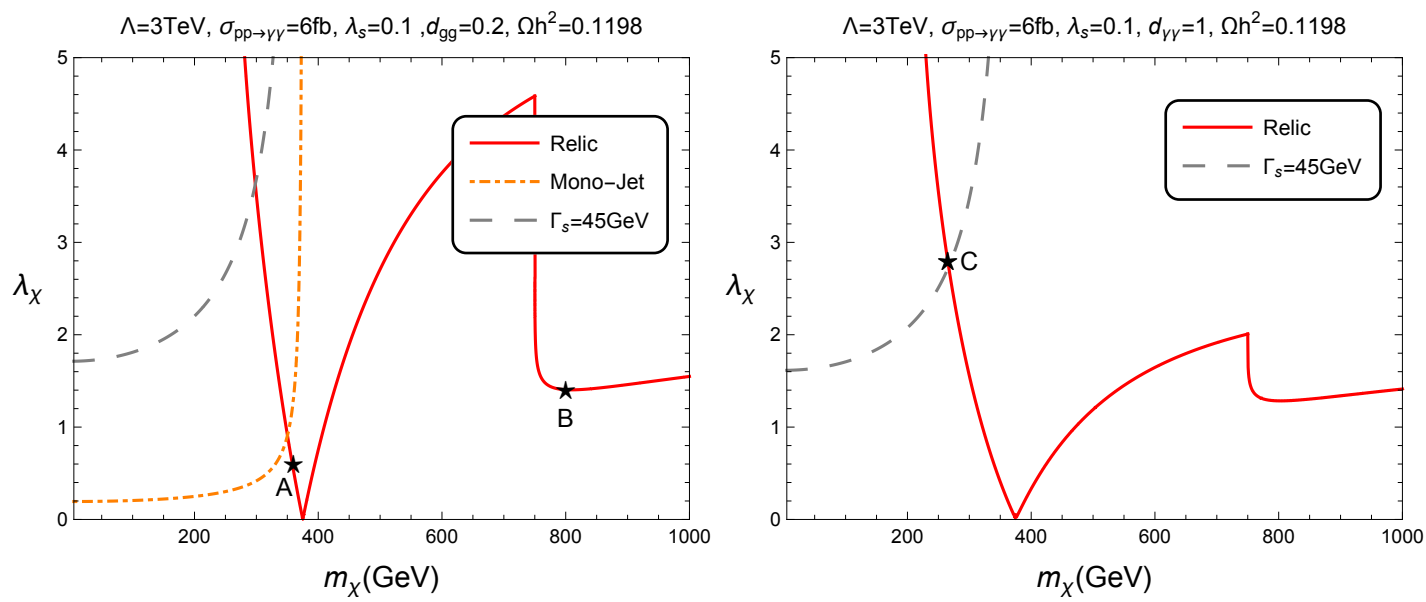

Figure 8. Parameter space of $m_{\chi}$ and $\lambda_{\chi}$ with real-scalar mediator, satisfying the relic density in red lines. The region explaining the diphoton resonance at $750 \mathrm{GeV}$ for $\sigma(p p \rightarrow \gamma \gamma)=6 \mathrm{fb}$ is imposed. $d_{g g}=0.2$ and $d_{\gamma \gamma}=1.0$ are chosen on left and right, while $c_{2}=0$ in both plots. The mono-jet bound rom $\mathrm{LHC} 8 \mathrm{TeV}$ is shown in orange dot-dashed line. The line for $\Gamma_{s}=45 \mathrm{GeV}$ is also shown in dashed gray. Benchmark models A and B (C) are shown in star on the left (right) plot, taken in table 2 .

become

$$
\begin{aligned}
\left(\sigma v_{\mathrm{rel}}\right)_{s s}= & \frac{\lambda_{\chi}^{2}}{32 \pi}\left[\frac{\lambda_{\chi}^{2} m_{\chi}^{2}\left(2\left(m_{s}^{2}-2 m_{\chi}^{2}\right)^{2}+m_{\chi}^{4}\right)}{3\left(m_{s}^{2}-2 m_{\chi}^{2}\right)^{4}}+\frac{9 \lambda_{S}^{2} v_{s}^{2}}{2\left[\left(4 m_{\chi}^{2}-m_{s}^{2}\right)^{2}+\Gamma_{s}^{2} m_{s}^{2}\right]}\right. \\
& \left.+\frac{6 \sqrt{2} \lambda_{\chi} \lambda_{S} v_{s} m_{\chi}}{m_{s}^{2}-2 m_{\chi}^{2}} \frac{4 m_{\chi}^{2}-m_{s}^{2}}{\left(4 m_{\chi}^{2}-m_{s}^{2}\right)^{2}+\Gamma_{s}^{2} m_{s}^{2}}\right]\left(1-\frac{m_{s}^{2}}{m_{\chi}^{2}}\right)^{1 / 2} v_{\mathrm{rel}}^{2},
\end{aligned}
$$

Thus, the ss channel turns out to be $p$-wave suppressed, but it can contribute to the thermal cross section at freeze-out.

In figure 8 , we show the parameter space of dark matter mass $m_{\chi}$ and coupling $\lambda_{\chi}$ in the model with real-scalar resonance where the condition for diphoton excesses is satisfied. Depending on whether the gluon or photon coupling is dominant, namely, $d_{g g}=0.2\left(d_{\gamma \gamma}=\right.$ 1.0) on left (right) plots, respectively, with the photon or gluon coupling $\left(d_{\gamma \gamma}\right.$ or $\left.d_{g g}\right)$ being determined by the diphoton condition (3.3), we imposed the current bounds from monojet searches. In the former case with a large gluon coupling, the mono-jet bound is still strong below resonance, as in the case with pseudo-scalar resonance. But, in the latter case with a large photon coupling and accordingly a small gluon coupling due to the diphoton condition, there is no bound from mono-jet searches. As the dark matter annihilation into a pair of the SM particles in the s-channels or into a pair of CP-even scalars are p-wave suppressed, there is no bound from indirect detection on these models.

In table 5, we show the averaged annihilation cross sections at freeze-out and the relic density for dark matter with real-scalar mediator in some benchmark models considered in table 1, having passed the diphoton condition as well as the above current collider bounds. Models A and B (C) belong to the left(right) plot in figure 8. 


\begin{tabular}{|c||c|c|c|c|c|c|}
\hline Model & $\left\langle\sigma v_{\text {rel }}\right\rangle_{s, \gamma \gamma}$ & $\left\langle\sigma v_{\text {rel }}\right\rangle_{s, g g}$ & $\left\langle\sigma v_{\text {rel }}\right\rangle_{s, Z \gamma}$ & $\left\langle\sigma v_{\text {rel }}\right\rangle_{s, Z Z}$ & $\left\langle\sigma v_{\text {rel }}\right\rangle_{s s}$ & $\Omega_{\chi} h^{2}$ \\
\hline A & $2.79 \times 10^{-29}$ & $4.95 \times 10^{-26}$ & $1.61 \times 10^{-29}$ & $2.28 \times 10^{-30}$ & - & 0.116 \\
\hline $\mathrm{B}$ & $2.12 \times 10^{-30}$ & $3.99 \times 10^{-27}$ & $1.27 \times 10^{-30}$ & $1.87 \times 10^{-31}$ & $4.15 \times 10^{-26}$ & 0.121 \\
\hline $\mathrm{C}$ & $2.97 \times 10^{-26}$ & $6.44 \times 10^{-29}$ & $1.61 \times 10^{-26}$ & $2.08 \times 10^{-27}$ & - & 0.120 \\
\hline
\end{tabular}

Table 5. Averaged annihilation cross sections (in units of $\mathrm{cm}^{3} / \mathrm{s}$ ) at freeze-out and relic density for dark matter with real-scalar. The benchmark models are the same as in table 2 and figure 8 . All the constraints from the current collider and cosmic data are satisfied.

\section{Dark matter with pseudo- and real-scalars}

In this section, we consider alternative interpretations of the diphoton excess as the degenerate real- and pseudo-scalar resonances or the cascade decay of the real-scalar into a pair of pseudo-scalars, each of which decays into a pair of photons. In these cases, we incorporate the constraints from dark matter and collider searches in the model.

\subsection{Dark matter annihilation}

When both pseudo-scalar and real scalar are included in the effective field theory, either or both of them can produce the diphoton resonance and contribute to the annihilation of dark matter.

For $m_{\chi}>\left(m_{a}+m_{s}\right) / 2$, the dark matter annihilation into as is open as shown in figure 9 and it gives rise to an additional annihilation cross section of dark matter, given by

$$
\begin{aligned}
\left(\sigma v_{\mathrm{rel}}\right)_{a s}= & \frac{\lambda_{\chi}^{2}}{64 \pi m_{\chi}^{2}}\left[\frac{\lambda_{\chi}^{2}\left(4 m_{\chi}^{2}-m_{s}^{2}+m_{a}^{2}\right)^{2}}{\left(m_{s}^{2}+m_{a}^{2}-4 m_{\chi}^{2}\right)^{2}}+\frac{8 \lambda_{S}^{2} v_{s}^{2} m_{\chi}^{2}}{\left(4 m_{\chi}^{2}-m_{a}^{2}\right)^{2}+\Gamma_{a}^{2} m_{a}^{2}}\right. \\
& \left.+\frac{4 \sqrt{2} \lambda_{\chi} \lambda_{S} v_{s} m_{\chi}\left(4 m_{\chi}^{2}-m_{a}^{2}\right)}{\left(4 m_{\chi}^{2}-m_{a}^{2}\right)^{2}+\Gamma_{a}^{2} m_{a}^{2}} \cdot \frac{4 m_{\chi}^{2}-m_{s}^{2}+m_{a}^{2}}{m_{s}^{2}+m_{a}^{2}-4 m_{\chi}^{2}}\right] \\
& \times\left(1-\frac{\left(m_{s}-m_{a}\right)^{2}}{4 m_{\chi}^{2}}\right)^{1 / 2}\left(1-\frac{\left(m_{s}+m_{a}\right)^{2}}{4 m_{\chi}^{2}}\right)^{1 / 2} .
\end{aligned}
$$

We can see that the as channel is s-wave so it is also relevant for indirect detection at present. In this case, the total annihilation cross section of dark matter is given by $\left(\sigma v_{\text {rel }}\right)_{\text {tot }}=\left(\sigma v_{\text {rel }}\right)_{a}+\left(\sigma v_{\text {rel }}\right)_{s}+\left(\sigma v_{\text {rel }}\right)_{a s}$, where the first two contributions are given in the previous sections with a single scalar resonance.

First, when two singlet scalars are almost degenerate in mass, namely, $m_{a} \approx m_{s} \approx$ $750 \mathrm{GeV}$, they both contribute to the diphoton excesses. In this case, the new as annihilation channel of dark matter is open only for a heavy dark matter with $m_{\chi} \gtrsim 750 \mathrm{GeV}$.

On the other hand, when the pseudo-scalar or real-scalar is light enough, $m_{a} \lesssim$ $0.4 \mathrm{GeV} \ll m_{s}=750 \mathrm{GeV}$ or $m_{s} \lesssim 0.4 \mathrm{GeV} \ll m_{a}=750 \mathrm{GeV}$, we can identify the real scalar or pseudo-scalar as the diphoton resonance and obtain the diphoton excess from the cascade decay of the real scalar $(s \rightarrow a a$ with $a \rightarrow \gamma \gamma)$ in the former case or the direct decay of the pseudo-scalar $(a \rightarrow \gamma \gamma)$ in the latter case. In these cases, the as annihilation channel of dark matter is open even for a relatively light dark matter with $m_{\chi} \gtrsim 375 \mathrm{GeV}$. 


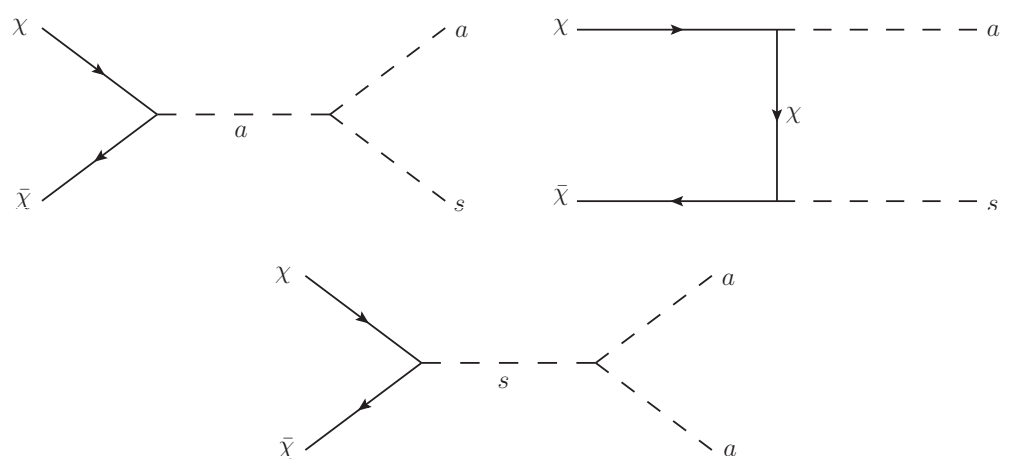

Figure 9. Feynman diagrams for dark matter annihilation into a pair of real- and pseudo-scalars and an additional channel with real-scalar mediator.

For our discussion, we focus on the former case when the pseudo-scalar is much lighter than the real-scalar, as it is natural for a small soft breaking of the U(1) global symmetry.

\subsection{Indirect detection}

As the pseudo-scalar is light, it mediates dark matter annihilations. In particular, dark matter annihilation channels, $\chi \bar{\chi} \rightarrow a \rightarrow \gamma \gamma, Z \gamma$, are s-wave and they lead to monochromatic photons at $E_{\gamma}=m_{\chi}$ and $E_{\gamma}=m_{\chi}\left(1-\frac{4 m_{Z}^{2}}{m_{\chi}^{2}}\right)$, respectively, as in the case with the pseudo-scalar resonance, so the model can be constrained by Ferm-LAT [75-78] and HESS [82] line searches.

Furthermore, annihilation channels of dark matter into $W W, Z Z, g g$ in s-channels with pseudo-scalar or the annihilation channel, $\chi \bar{\chi} \rightarrow a s$ with $s \rightarrow W W, Z Z, g g$ and/or $a \rightarrow g g$ lead to continuum photons from bremstrahlung or decay and they are constrained by Fermi-diffuse gamma-ray searches from dwarf galaxies [79-81]. Moreover, dark matter annihilation into gluons can be constrained by anti-proton data from PAMELA and AMS02 [83-86]. Since the pseudo-scalar has sub-GeV mass, the $s$-wave annihilation of weakscale dark matter is not enhanced due to a resonance, but rather it gets smaller as dark matter increases. Furthermore, small effective couplings of scalars are allowed in the case of cascade decay. Therefore, the indirect bounds on the $s$-wave channels are weaker than the case with pseudo-scalar resonance. In this case, the $p$-wave annihilation of dark matter with real-scalar resonance becomes important at freeze-out, determining the relic density.

In the presence of a light pseudo-scalar, there is an additional $s$-wave annihilation channel, $\chi \bar{\chi} \rightarrow a s$, is $s$-wave, and it leads to multi-photons due to the direct decay $a \rightarrow \gamma \gamma$ or the cascade decay of the real scalar, $s \rightarrow a a$, with $a \rightarrow \gamma \gamma$. The gamma-ray boxes could be constrained further by line-like features in Fermi-LAT and HESS, leading to more stringent bounds than Fermi-LAT diffuse gamma-ray searches or anti-proton searches, depending on the branching fractions of scalars.

We briefly discuss the gamma-ray energy obtained from $\chi \bar{\chi} \rightarrow$ as channel. The decay $a \rightarrow \gamma \gamma$ produces two photons with identical energy in the rest frame of the pseudo-scalar, $E_{\gamma}^{*}=m_{a} / 2$. However, in the galactic frame, where the dark matter particles move non- 
relativistically, the photon energy reads $[61,87]$

$$
E_{\gamma}=\frac{1}{\gamma_{a}} E_{\gamma}^{*}\left(1-v_{a} \cos \theta_{a}\right)^{-1},
$$

where $\gamma_{a} \equiv 1 / \sqrt{1-v_{a}^{2}}, \theta_{a}$ is the angle between the direction of the pseudo-scalar and the direction of the photon and $v_{a}$ is the pseudo-scalar velocity, given by

$$
v_{a}=\frac{p_{a}}{E_{a}}=\sqrt{1-\frac{m_{a}^{2}}{m_{\chi}^{2}}\left(1+\frac{m_{a}^{2}-m_{s}^{2}}{4 m_{\chi}^{2}}\right)^{-2}} .
$$

Since the pseudo-scalar decays isotropically, the resulting energy spectrum presents a boxshaped structure with the photon energy ranging from $E_{-}$to $E_{+}$, where $E_{ \pm}=\frac{1}{2} A m_{\chi}(1 \pm$ $\left.\sqrt{1-\frac{m_{a}^{2}}{A^{2} m_{\chi}^{2}}}\right)$ and $A=1+\left(m_{a}^{2}-m_{s}^{2}\right) /\left(4 m_{\chi}^{2}\right)$. Then, the energy spectrum of two hard photons produced in the annihilation channel $\chi \bar{\chi} \rightarrow$ as [61] is

$$
\frac{d N_{\gamma}^{(2)}}{d E_{\gamma}}=\frac{2}{E_{+}-E_{-}} \Theta\left(E_{\gamma}-E_{-}\right) \Theta\left(E_{+}-E_{\gamma}\right) \operatorname{BR}(a \rightarrow \gamma \gamma)
$$

where $\Theta$ is the Heaviside function.

Furthermore, the cascade decay $s \rightarrow a a \rightarrow 4 \gamma$ with a large $\operatorname{BR}(a \rightarrow \gamma \gamma)$ leads to four additional photons, thus leading to potentially interesting signatures in gamma-ray searches, which will be published elsewhere. In this work, we focus on the box-shaped gamma-ray spectrum to get a conservative bound on the annihilation cross section for $\chi \bar{\chi} \rightarrow a s$.

In figure 10, we show the parameter space of dark matter mass $m_{\chi}$ and coupling $\lambda_{\chi}$ in the model with $m_{s}=750 \mathrm{GeV}$ and $m_{a}=0.4 \mathrm{GeV}$ where the condition for diphoton excesses is satisfied. We have set $c_{2}=0$ for simplicity. Depending on the value of the gluon coupling $c_{g g}=0.1(0.01)$ in the upper and lower panels, respectively, with the photon coupling $c_{\gamma \gamma}$ being determined by the diphoton condition (3.3), or the quartic coupling of the complex scalar, $\lambda_{S}=0.1(1.0)$ on left and right in each panel, respectively, we imposed the current bounds from mono-jet searches as well as various indirect detections.

First, on the left plot in the upper panel with $c_{\gamma \gamma}=\lambda_{S}=0.1$ in figure 10, the monojet bound excludes most of the region below resonance, while Fermi-LAT line and other indirect searches are not sensitive enough yet to constrain the region with saturated relic density. When the photon coupling gets smaller but the quartic coupling remains small as on the left plot in the lower panel with $c_{\gamma \gamma}=0.05$ and $\lambda_{S}=0.1$, the Fermi-LAT line search does not constrain the region below resonance. The important difference from the case with pseudo-scalar resonance is that there appears a bound from the Fermi-LAT search for gamma-ray box, although it is not sensitive enough yet to the region with saturated relic density. Finally, when the quartic coupling gets larger to $\lambda_{S}=1.0$ as in the right plots with $c_{\gamma \gamma}=0.1$ or 0.05 , the diphoton excesses can be explained dominantly by the cascade decay of real-scalar. In these cases, either mono-jet or Fermi-LAT searches do not reach the region with saturated relic density, opening up more parameter space to be probed for in the LHC Run 2 and future gamma-ray searches such as Cherenkov Telescope Array [88]. 

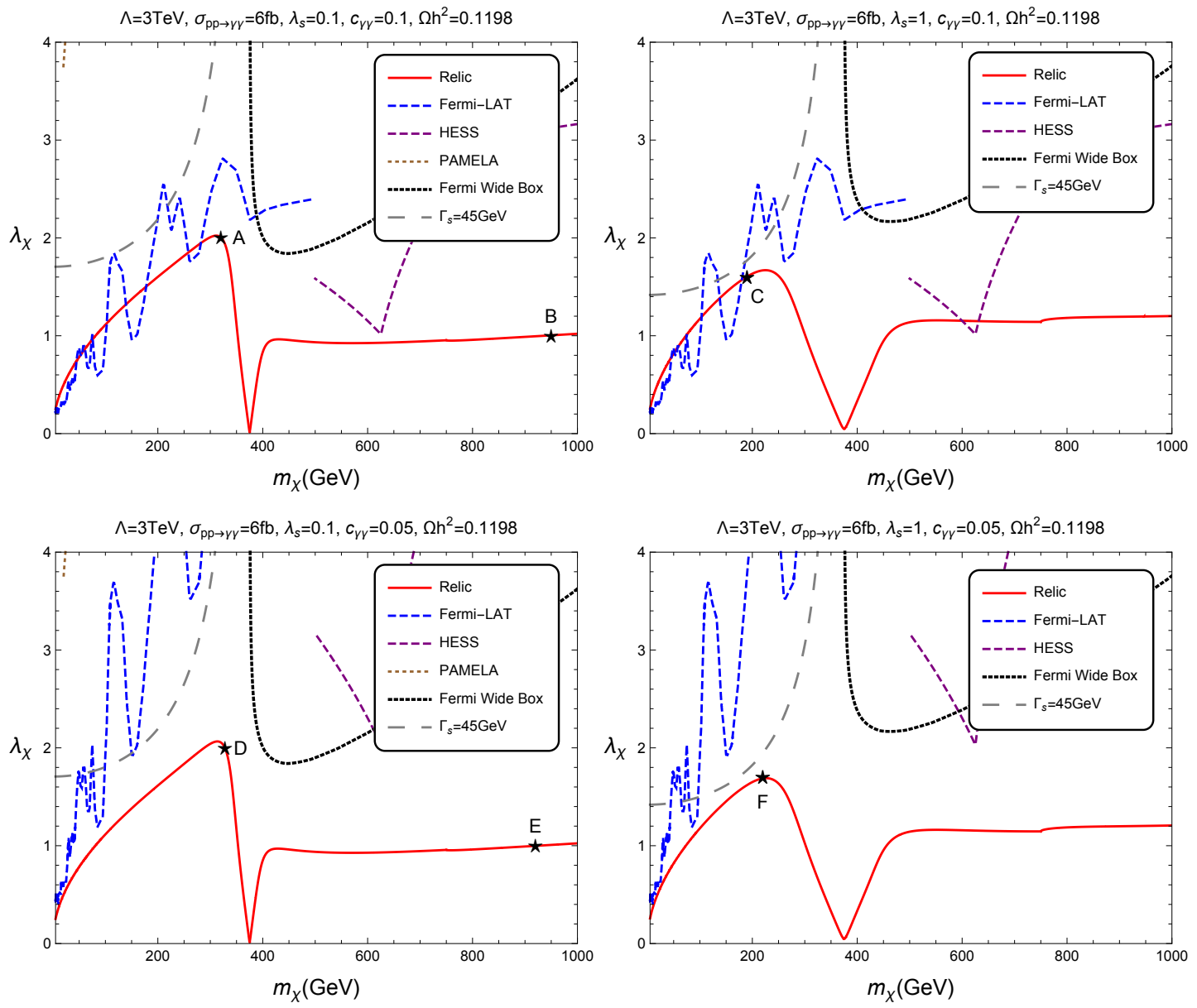

Figure 10. Parameter space of $m_{\chi}$ and $\lambda_{\chi}$ with both direct and cascade decays of the real scalar, satisfying the relic density in red lines. The condition explaining the diphoton resonance at $750 \mathrm{GeV}$ for $\sigma(p p \rightarrow \gamma \gamma)=6 \mathrm{fb}$ is imposed. We took $m_{s}=750 \mathrm{GeV}$ and $m_{a}=0.4 \mathrm{GeV}$. $c_{\gamma \gamma}=0.1$ and $\lambda_{S}=0.1(1.0)$ are chosen on left (right) in the upper panel while $c_{\gamma \gamma}=0.05$ and $\lambda_{S}=0.1(1.0)$ are chosen on left (right) in the lower panel. We have taken $c_{2}=0$ in all plots. The mono-jet limit from LHC $8 \mathrm{TeV}$ and the limits from Fermi-LAT, PAMELA and HESS are shown in orange dot-dashed, blue dashed, brown dotted, and purple dashed lines, respectively. The limits from AMS-02 and Fermi-LAT wide box are also shown in pink dotted and black dotted lines. The line for $\Gamma_{s}=45 \mathrm{GeV}$ is also shown in dashed gray. Benchmark models $\mathrm{A}$ and $\mathrm{B}(\mathrm{C})$ are shown in star on the left(right) plot in the upper panel, while models D and E (F) are shown in star on the left(right) plot in the lower panel. Those benchmark models are taken in table 3.

In table 6 , we show the averaged annihilation cross sections for $s$-wave channels such as $\gamma \gamma, g g, Z \gamma, Z Z$, as, at present, except those for $a a, s s$ channels, which are taken at freezeout, and the relic density for dark matter with two scalar fields in some benchmark models considered in table 3. The diphoton condition as well as the above current collider bounds are satisfied for all the models in table 6 . We have set the scalar masses to $m_{s}=750 \mathrm{GeV}$ and $m_{a}=0.4 \mathrm{GeV}$ and the effective gauge couplings to $d_{i}=\frac{4}{3} c_{i}(i=1,3)$ and $c_{2}=d_{2}=0$. Models A and B (C) belong to the left(right) plot in the upper panel of figure 10, while 


\begin{tabular}{|c||c|c|c|c|}
\hline Model & $\left\langle\sigma v_{\text {rel }}\right\rangle_{\gamma \gamma}$ & $\left\langle\sigma v_{\text {rel }}\right\rangle_{g g}$ & $\left\langle\sigma v_{\text {rel }}\right\rangle_{Z \gamma}$ & $\left\langle\sigma v_{\text {rel }}\right\rangle_{Z Z}$ \\
\hline $\mathrm{A}$ & $2.06 \times 10^{-27}$ & $1.25 \times 10^{-28}$ & $1.17 \times 10^{-27}$ & $1.64 \times 10^{-28}$ \\
\hline $\mathrm{B}$ & $5.16 \times 10^{-28}$ & $4.98 \times 10^{-30}$ & $3.10 \times 10^{-28}$ & $4.58 \times 10^{-29}$ \\
\hline $\mathrm{C}$ & $1.32 \times 10^{-27}$ & $3.27 \times 10^{-29}$ & $6.69 \times 10^{-28}$ & $8.03 \times 10^{-29}$ \\
\hline $\mathrm{D}$ & $5.16 \times 10^{-28}$ & $1.07 \times 10^{-28}$ & $2.95 \times 10^{-28}$ & $4.12 \times 10^{-29}$ \\
\hline $\mathrm{E}$ & $1.29 \times 10^{-28}$ & $4.88 \times 10^{-30}$ & $7.75 \times 10^{-29}$ & $1.14 \times 10^{-29}$ \\
\hline $\mathrm{F}$ & $3.73 \times 10^{-28}$ & $3.55 \times 10^{-29}$ & $1.98 \times 10^{-28}$ & $2.53 \times 10^{-29}$ \\
\hline
\end{tabular}

\begin{tabular}{|c||c|c|c|c|}
\hline Model & $\left\langle\sigma v_{\text {rel }}\right\rangle_{a a}$ & $\left\langle\sigma v_{\text {rel }}\right\rangle_{a s}$ & $\left\langle\sigma v_{\text {rel }}\right\rangle_{s s}$ & $\Omega_{\chi} h^{2}$ \\
\hline A & $8.47 \times 10^{-26}$ & - & - & 0.121 \\
\hline B & $1.10 \times 10^{-27}$ & $4.16 \times 10^{-26}$ & $3.19 \times 10^{-27}$ & 0.122 \\
\hline C & $9.14 \times 10^{-26}$ & - & - & 0.121 \\
\hline D & $9.31 \times 10^{-26}$ & - & - & 0.119 \\
\hline $\mathrm{E}$ & $1.18 \times 10^{-27}$ & $4.34 \times 10^{-26}$ & $3.45 \times 10^{-27}$ & 0.119 \\
\hline $\mathrm{F}$ & $9.76 \times 10^{-26}$ & - & - & 0.117 \\
\hline
\end{tabular}

Table 6. Averaged annihilation cross sections (in units of $\mathrm{cm}^{3} / \mathrm{s}$ ) at present and relic density for dark matter with two scalars, except that those for $a a, s s$ channels are given at freeze-out. The benchmark models are the same as in table 3 and figure 10. All the constraints from the current collider and cosmic data are satisfied.

Model E and D (F) belongs to the left(right) plot in the lower panel of figure 10. These models satisfy the current bounds from various indirect detection experiments discussed above. As the $a a, s s$ channels are $p$-wave suppressed and negligible at present, there is no bound on those channels from indirect detection. So, we show in the same table the annihilation cross sections for the $a a, s s$ channels at freeze-out.

\section{Conclusions}

We have considered various possibilities of explaining the diphoton excesses observed at the LHC in terms of singlet scalar resonances with effective interactions to gluons and photon. In the case that the resonance decays directly into a photon pair, the region of the parameter space where there is an invisible decay of the resonance into a pair of dark matter particles is strongly constrained by the interplay between mono-jet and Fermi-LAT gamma-ray searches. When the diphoton excesses stem from the cascade decay of the real-scalar into a pair of pseudo-scalars, the effective couplings for SM gauge bosons can be smaller. In this case, the collider and indirect detection bounds are less strong, but the gamma-ray box coming from the cascade annihilation of dark matter into a pair of realscalar and pseudo-scalar could be a smoking-gun signal in gamma-ray searches. We have shown various benchmark models that are consistent with all the collider and astrophysical constraints and can be testable in the LHC Run 2 as well as future gamma-ray searches. 


\section{Acknowledgments}

The work of HML is supported in part by Basic Science Research Program through the National Research Foundation of Korea (NRF) funded by the Ministry of Education, Science and Technology (2013R1A1A2007919). The work of YJK is supported by the Chung-Ang University Graduate Research Scholarship in 2016.

\section{A Scalar sector of the model}

In the text, we consider the scalar potential for the singlet complex scalar $S$ and the SM Higgs doublet $H$ is

$$
V(H, S)=\lambda_{H}|H|^{4}+\lambda_{S}|S|^{4}+2 \lambda_{H S}|S|^{2}|H|^{2}+m_{H}^{2}|H|^{2}+m_{S}^{2}|S|^{2}-\left(\frac{1}{2} m_{S}^{\prime 2} S^{2}+\text { h.c. }\right)
$$

where $m_{S}^{\prime}$ term breaks the U(1) global symmetry softly to give the pseudo-scalar component mass. After minimizing the potential in eq. (A.1), the VEVs of the singlet and the Higgs doublet, $v_{s}$ and $v$, are determined as

$$
\begin{aligned}
& v_{s}^{2}=\frac{\lambda_{H S} m_{H}^{2}-\lambda_{H}\left(m_{S}^{2}-m_{S}^{\prime 2}\right)}{\lambda_{S} \lambda_{H}-\lambda_{H S}^{2}}, \\
& v^{2}=\frac{\lambda_{H S}\left(m_{S}^{2}-m_{S}^{2}\right)-\lambda_{S} m_{H}^{2}}{\lambda_{S} \lambda_{H}-\lambda_{H S}^{2}} .
\end{aligned}
$$

The conditions for a local minimum are $\lambda_{H S} m_{H}^{2}-\lambda_{H}\left(m_{S}^{2}-m_{S}^{\prime 2}\right)>0, \lambda_{H S}\left(m_{S}^{2}-m_{S}^{\prime 2}\right)-$ $\lambda_{S} m_{H}^{2}>0$ and $\lambda_{S} \lambda_{H}-\lambda_{H S}^{2}>0$. Expanding the scalar fields around the vacuum as $S=\left(v_{s}+s+i a\right) / \sqrt{2}$ and $H^{T}=(0, v+h) / \sqrt{2}$ in unitary gauge, the obtained mass matrix for $\mathrm{CP}$-even scalars can be diagonalized by the field rotation,

$$
s=\cos \theta h_{2}+\sin \theta h_{1}, \quad h=-\sin \theta h_{2}+\cos \theta h_{1}
$$

with

$$
\tan 2 \theta=\frac{2 \lambda_{H S} v_{s} v}{\lambda_{H} v^{2}-\lambda_{S} v_{s}^{2}}
$$

and the mass eigenvalues are

$$
m_{1,2}^{2}=\lambda_{H} v^{2}+\lambda_{S} v_{s}^{2} \mp \sqrt{\left(\lambda_{S} v_{s}^{2}-\lambda_{H} v^{2}\right)^{2}+4 \lambda_{H S}^{2} v^{2} v_{s}^{2}} .
$$

Thus, $h_{1}$ is Higgs-like and $h_{2}$ is singlet-like.

We also note that the singlet-like scalar $h_{2}$ can have Higgs-like couplings to the SM particles through the mixing with the Higgs boson as well as scalar triple self-couplings given by

$$
\begin{aligned}
\mathcal{L}_{\text {scalar }}= & c_{h_{1} a a} h_{1} a^{2}+c_{h_{2} a a} h_{2}^{2} a^{2} \\
& +c_{h_{1} h_{1} h_{1}} h_{1}^{3}+c_{h_{2} h_{2} h_{2}} h_{2}^{3}+c_{h_{1} h_{2} h_{2}} h_{1} h_{2}^{2}+c_{h_{1} h_{1} h_{2}} h_{1}^{2} h_{2}
\end{aligned}
$$


where

$$
\begin{aligned}
c_{h_{1} a a}= & -\lambda_{H S} v \cos \theta-\lambda_{S} v_{s} \sin \theta \\
c_{h_{2} a a}= & \lambda_{H S} v \sin \theta-\lambda_{S} v_{s} \cos \theta \\
c_{h_{1} h_{1} h_{1}}= & -\lambda_{H} v \cos ^{3} \theta-\lambda_{H S} \cos \theta \sin \theta\left(v \sin \theta+v_{s} \cos \theta\right)-\lambda_{S} v_{s} \sin ^{3} \theta, \\
c_{h_{2} h_{2} h_{2}}= & \lambda_{H} v \sin ^{3} \theta+\lambda_{H S} \cos \theta \sin \theta\left(v \cos \theta-v_{s} \sin \theta\right)-\lambda_{S} v_{s} \cos ^{3} \theta \\
c_{h_{1} h_{2} h_{2}}= & -3 \lambda_{H} v \sin ^{2} \theta \cos \theta-\lambda_{H S}\left(v \cos \theta\left(\cos ^{2} \theta-2 \sin ^{2} \theta\right)\right. \\
& \left.+v_{s} \sin \theta\left(\sin ^{2} \theta-2 \cos ^{2} \theta\right)\right)-3 \lambda_{S} v_{s} \sin \theta \cos ^{2} \theta \\
c_{h_{1} h_{1} h_{2}}= & 3 \lambda_{H} v \cos ^{2} \theta \sin \theta+\lambda_{H S}\left(v \sin \theta\left(\sin ^{2} \theta-2 \cos ^{2} \theta\right)\right. \\
& \left.-v_{s} \cos \theta\left(\cos ^{2} \theta-2 \sin ^{2} \theta\right)\right)-3 \lambda_{S} v_{s} \cos \theta \sin ^{2} \theta .
\end{aligned}
$$

Open Access. This article is distributed under the terms of the Creative Commons Attribution License (CC-BY 4.0), which permits any use, distribution and reproduction in any medium, provided the original author(s) and source are credited.

\section{References}

[1] ATLAS collaboration, Search for resonances decaying to photon pairs in 3.2 $\mathrm{fb}^{-1}$ of $\mathrm{pp}$ collisions at $\sqrt{s}=13 \mathrm{TeV}$ with the ATLAS detector, ATLAS-CONF-2015-081 (2015).

[2] CMS collaboration, Search for new physics in high mass diphoton events in proton-proton collisions at $\sqrt{s}=13 \mathrm{TeV}$, CMS-PAS-EXO-15-004 (2015).

[3] ATLAS collaboration, Search for resonances in diphoton events with the ATLAS detector at $\sqrt{s}=13 \mathrm{Te} V$, ATLAS-CONF-2016-018 (2016).

[4] CMS collaboration, Search for new physics in high mass diphoton events in $3.3 \mathrm{fb}^{-1}$ of proton-proton collisions at $\sqrt{s}=13 \mathrm{TeV}$ and combined interpretation of searches at $8 \mathrm{TeV}$ and $13 \mathrm{TeV}$, CMS-PAS-EXO-16-018 (2016).

[5] R. Franceschini et al., What is the $\gamma \gamma$ resonance at 750 GeV?, JHEP 03 (2016) 144 [arXiv: 1512.04933] [INSPIRE].

[6] R. Franceschini et al., Digamma, what next?, arXiv:1604.06446 [INSPIRE].

[7] J. Ellis, S.A.R. Ellis, J. Quevillon, V. Sanz and T. You, On the Interpretation of a Possible $\sim 750 \mathrm{GeV}$ Particle Decaying into $\gamma \gamma$, JHEP 03 (2016) 176 [arXiv:1512.05327] [INSPIRE].

[8] A. Falkowski, O. Slone and T. Volansky, Phenomenology of a $750 \mathrm{GeV}$ Singlet, JHEP 02 (2016) 152 [arXiv : 1512.05777] [INSPIRE].

[9] J.S. Kim, K. Rolbiecki and R. Ruiz de Austri, Model-independent combination of diphoton constraints at $750 \mathrm{GeV}$, Eur. Phys. J. C 76 (2016) 251 [arXiv: 1512.06797] [INSPIRE].

[10] M.R. Buckley, Wide or narrow? The phenomenology of $750 \mathrm{GeV}$ diphotons, Eur. Phys. J. C 76 (2016) 345 [arXiv: 1601.04751] [INSPIRE].

[11] J.F. Kamenik, B.R. Safdi, Y. Soreq and J. Zupan, Comments on the diphoton excess: critical reappraisal of effective field theory interpretations, arXiv:1603.06566 [INSPIRE].

[12] K. Harigaya and Y. Nomura, Composite Models for the 750 GeV Diphoton Excess, Phys. Lett. B 754 (2016) 151 [arXiv:1512.04850] [INSPIRE]. 
[13] A. Pilaftsis, Diphoton Signatures from Heavy Axion Decays at the CERN Large Hadron Collider, Phys. Rev. D 93 (2016) 015017 [arXiv:1512.04931] [InSPIRE].

[14] T. Higaki, K.S. Jeong, N. Kitajima and F. Takahashi, The QCD Axion from Aligned Axions and Diphoton Excess, Phys. Lett. B 755 (2016) 13 [arXiv:1512.05295] [INSPIRE].

[15] J.M. No, V. Sanz and J. Setford, See-saw composite Higgs model at the LHC: Linking naturalness to the $750 \mathrm{GeV}$ diphoton resonance, Phys. Rev. D 93 (2016) 095010 [arXiv: 1512.05700] [INSPIRE].

[16] A. Belyaev, G. Cacciapaglia, H. Cai, T. Flacke, A. Parolini and H. Serôdio, Singlets in Composite Higgs Models in light of the LHC di-photon searches, arXiv:1512.07242 [INSPIRE].

[17] L.J. Hall, K. Harigaya and Y. Nomura, 750 GeV Diphotons: Implications for Supersymmetric Unification, JHEP 03 (2016) 017 [arXiv:1512.07904] [INSPIRE].

[18] M. Son and A. Urbano, A new scalar resonance at $750 \mathrm{GeV}$ : Towards a proof of concept in favor of strongly interacting theories, JHEP 05 (2016) 181 [arXiv: 1512.08307] [INSPIRE].

[19] J.E. Kim, Is an axizilla possible for di-photon resonance?, Phys. Lett. B 755 (2016) 190 [arXiv: 1512.08467] [INSPIRE].

[20] A. Angelescu, A. Djouadi and G. Moreau, Scenarii for interpretations of the LHC diphoton excess: two Higgs doublets and vector-like quarks and leptons, Phys. Lett. B 756 (2016) 126 [arXiv: 1512.04921] [INSPIRE].

[21] S. Di Chiara, L. Marzola and M. Raidal, First interpretation of the $750 \mathrm{GeV}$ diphoton resonance at the LHC, Phys. Rev. D 93 (2016) 095018 [arXiv: 1512.04939] [INSPIRE].

[22] W. Altmannshofer, J. Galloway, S. Gori, A.L. Kagan, A. Martin and J. Zupan, $750 \mathrm{GeV}$ diphoton excess, Phys. Rev. D 93 (2016) 095015 [arXiv:1512.07616] [INSPIRE].

[23] P. Ko, Y. Omura and C. Yu, Diphoton Excess at $750 \mathrm{GeV}$ in leptophobic U(1)' model inspired by $E_{6}$ GUT, JHEP 04 (2016) 098 [arXiv: 1601.00586] [INSPIRE].

[24] T. Modak, S. Sadhukhan and R. Srivastava, $750 \mathrm{GeV}$ diphoton excess from gauged $B-L$ symmetry, Phys. Lett. B 756 (2016) 405 [arXiv:1601.00836] [InSPIRE].

[25] C.-W. Chiang, H. Fukuda, M. Ibe and T.T. Yanagida, $750 \mathrm{GeV}$ diphoton resonance in a visible heavy QCD axion model, Phys. Rev. D 93 (2016) 095016 [arXiv:1602.07909] [INSPIRE].

[26] C. Bonilla, M. Nebot, R. Srivastava and J.W.F. Valle, Flavor physics scenario for the $750 \mathrm{GeV}$ diphoton anomaly, Phys. Rev. D 93 (2016) 073009 [arXiv:1602.08092] [INSPIRE].

[27] H.P. Nilles and M.W. Winkler, $750 \mathrm{GeV}$ Diphotons and Supersymmetric Grand Unification, JHEP 05 (2016) 182 [arXiv: 1604.03598] [INSPIRE].

[28] K. Choi, S.H. Im, H. Kim and D.Y. Mo, $750 \mathrm{GeV}$ diphoton resonance and electric dipole moments, arXiv: 1605.00206 [INSPIRE].

[29] A. Djouadi and A. Pilaftsis, The 750 GeV Diphoton Resonance in the MSSM, arXiv: 1605.01040 [INSPIRE].

[30] C. Han, H.M. Lee, M. Park and V. Sanz, The diphoton resonance as a gravity mediator of dark matter, Phys. Lett. B 755 (2016) 371 [arXiv:1512.06376] [INSPIRE]. 
[31] S.B. Giddings and H. Zhang, Kaluza-Klein graviton phenomenology for warped compactifications and the $750 \mathrm{GeV}$ diphoton excess, Phys. Rev. D 93 (2016) 115002 [arXiv: 1602.02793] [INSPIRE].

[32] A. Falkowski and J.F. Kamenik, Di-photon portal to warped gravity, arXiv:1603.06980 [INSPIRE].

[33] J.L. Hewett and T.G. Rizzo, 750 GeV Diphoton Resonance in Warped Geometries, arXiv: 1603.08250 [INSPIRE].

[34] B.M. Dillon and V. Sanz, A Little KK Graviton at $750 \mathrm{GeV}$, arXiv:1603.09550 [INSPIRE].

[35] V. Sanz, Theoretical interpretation of a spin-two diphoton excess, arXiv:1603.05574 [INSPIRE].

[36] P. Roig and J.J. Sanz-Cillero, A broad $750 \mathrm{GeV}$ diphoton resonance? Not alone, arXiv: 1605.03831 [INSPIRE].

[37] Y. Mambrini, G. Arcadi and A. Djouadi, The LHC diphoton resonance and dark matter, Phys. Lett. B 755 (2016) 426 [arXiv: 1512.04913] [INSPIRE].

[38] M. Backovic, A. Mariotti and D. Redigolo, Di-photon excess illuminates Dark Matter, JHEP 03 (2016) 157 [arXiv: 1512.04917] [INSPIRE].

[39] X.-J. Bi, Q.-F. Xiang, P.-F. Yin and Z.-H. Yu, The $750 \mathrm{GeV}$ diphoton excess at the LHC and dark matter constraints, Nucl. Phys. B 909 (2016) 43 [arXiv: 1512.06787] [INSPIRE].

[40] U.K. Dey, S. Mohanty and G. Tomar, $750 \mathrm{GeV}$ resonance in the dark left-right model, Phys. Lett. B 756 (2016) 384 [arXiv:1512.07212] [INSPIRE].

[41] J.-C. Park and S.C. Park, Indirect signature of dark matter with the diphoton resonance at $750 \mathrm{GeV}$, arXiv: 1512.08117 [INSPIRE].

[42] S. Bhattacharya, S. Patra, N. Sahoo and N. Sahu, 750 GeV diphoton excess at CERN LHC from a dark sector assisted scalar decay, JCAP 06 (2016) 010 [arXiv:1601.01569] [INSPIRE].

[43] F. D'Eramo, J. de Vries and P. Panci, A $750 \mathrm{GeV}$ Portal: LHC Phenomenology and Dark Matter Candidates, JHEP 05 (2016) 089 [arXiv:1601.01571] [INSPIRE].

[44] P. Ko and T. Nomura, Dark sector shining through $750 \mathrm{GeV}$ dark Higgs boson at the LHC, Phys. Lett. B 758 (2016) 205 [arXiv: 1601.02490] [INSPIRE].

[45] A. Salvio, F. Staub, A. Strumia and A. Urbano, On the maximal diphoton width, JHEP 03 (2016) 214 [arXiv: 1602.01460] [INSPIRE].

[46] S.-F. Ge, H.-J. He, J. Ren and Z.-Z. Xianyu, Realizing Dark Matter and Higgs Inflation in Light of LHC Diphoton Excess, Phys. Lett. B 757 (2016) 480 [arXiv:1602.01801] [InSPIRE].

[47] M. Redi, A. Strumia, A. Tesi and E. Vigiani, Di-photon resonance and Dark Matter as heavy pions, JHEP 05 (2016) 078 [arXiv: 1602.07297] [INSPIRE].

[48] E. Morgante, D. Racco, M. Rameez and A. Riotto, The 750 GeV Diphoton excess, Dark Matter and Constraints from the IceCube experiment, arXiv:1603.05592 [INSPIRE].

[49] G. Arcadi, P. Ghosh, Y. Mambrini and M. Pierre, Re-opening dark matter windows compatible with a diphoton excess, arXiv:1603.05601 [INSPIRE].

[50] S. Knapen, T. Melia, M. Papucci and K. Zurek, Rays of light from the LHC, Phys. Rev. D 93 (2016) 075020 [arXiv: 1512.04928] [INSPIRE]. 
[51] P. Agrawal, J. Fan, B. Heidenreich, M. Reece and M. Strassler, Experimental Considerations Motivated by the Diphoton Excess at the LHC, JHEP 06 (2016) 082 [arXiv:1512.05775] [INSPIRE].

[52] J. Chang, K. Cheung and C.-T. Lu, Interpreting the $750 \mathrm{GeV}$ diphoton resonance using photon jets in hidden-valley-like models, Phys. Rev. D 93 (2016) 075013 [arXiv:1512.06671] [INSPIRE].

[53] L. Aparicio, A. Azatov, E. Hardy and A. Romanino, Diphotons from Diaxions, JHEP 05 (2016) 077 [arXiv : 1602.00949] [INSPIRE].

[54] U. Ellwanger and C. Hugonie, A $750 \mathrm{GeV}$ Diphoton Signal from a Very Light Pseudoscalar in the NMSSM, JHEP 05 (2016) 114 [arXiv:1602.03344] [INSPIRE].

[55] F. Domingo, S. Heinemeyer, J.S. Kim and K. Rolbiecki, The NMSSM lives: with the $750 \mathrm{GeV}$ diphoton excess, Eur. Phys. J. C 76 (2016) 249 [arXiv: 1602.07691] [INSPIRE].

[56] V. De Romeri, J.S. Kim, V. Martín-Lozano, K. Rolbiecki and R. Ruiz de Austri, Confronting dark matter with the diphoton excess from a parent resonance decay, Eur. Phys. J. C 76 (2016) 262 [arXiv : 1603.04479] [INSPIRE].

[57] Y. Jiang, L. Li and R. Zheng, Boosted scalar confronting 750 GeV di-photon excess, arXiv: 1605.01898 [INSPIRE].

[58] H.M. Lee, M. Park and W.-I. Park, Fermi Gamma Ray Line at $130 \mathrm{GeV}$ from Axion-Mediated Dark Matter, Phys. Rev. D 86 (2012) 103502 [arXiv:1205.4675] [InSPIRE].

[59] H.M. Lee, M. Park and W.-I. Park, Axion-mediated dark matter and Higgs diphoton signal, JHEP 12 (2012) 037 [arXiv: 1209.1955] [INSPIRE].

[60] H.M. Lee, M. Park and V. Sanz, Interplay between Fermi gamma-ray lines and collider searches, JHEP 03 (2013) 052 [arXiv: 1212.5647] [INSPIRE].

[61] A. Ibarra, H.M. Lee, S. López Gehler, W.-I. Park and M. Pato, Gamma-ray boxes from axion-mediated dark matter, JCAP 05 (2013) 016 [arXiv:1303.6632] [INSPIRE].

[62] X. Chu, T. Hambye, T. Scarna and M.H.G. Tytgat, What if Dark Matter Gamma-Ray Lines come with Gluon Lines?, Phys. Rev. D 86 (2012) 083521 [arXiv:1206. 2279] [INSPIRE].

[63] Y. Mambrini, S. Profumo and F.S. Queiroz, Dark Matter and Global Symmetries, arXiv:1508.06635 [INSPIRE].

[64] H.M. Lee et al., Discrete R symmetries for the MSSM and its singlet extensions, Nucl. Phys. B 850 (2011) 1 [arXiv: 1102.3595] [INSPIRE].

[65] K.-Y. Choi, E.J. Chun and H.M. Lee, Dark matter, mu problem and neutrino mass with gauged R-symmetry, Phys. Rev. D 82 (2010) 105028 [arXiv:1002.4791] [INSPIRE].

[66] H.M. Lee, M. Park and V. Sanz, Gravity-mediated (or Composite) Dark Matter Confronts Astrophysical Data, JHEP 05 (2014) 063 [arXiv:1401.5301] [INSPIRE].

[67] H.M. Lee, M. Park and V. Sanz, Gravity-mediated (or Composite) Dark Matter, Eur. Phys. J. C 74 (2014) 2715 [arXiv:1306.4107] [INSPIRE].

[68] ATLAS collaboration, Measurements of the Higgs boson production and decay rates and coupling strengths using pp collision data at $\sqrt{s}=7$ and $8 \mathrm{TeV}$ in the ATLAS experiment, Eur. Phys. J. C 76 (2016) 6 [arXiv:1507.04548] [InSPIRE]. 
[69] CMS collaboration, Precise determination of the mass of the Higgs boson and tests of compatibility of its couplings with the standard model predictions using proton collisions at 7 and 8 TeV, Eur. Phys. J. C 75 (2015) 212 [arXiv:1412.8662] [INSPIRE].

[70] ATLAS collaboration, Measurement of Higgs boson production in the diphoton decay channel in pp collisions at center-of-mass energies of 7 and 8 TeV with the ATLAS detector, Phys. Rev. D 90 (2014) 112015 [arXiv: 1408.7084] [INSPIRE].

[71] CMS collaboration, Observation of the diphoton decay of the Higgs boson and measurement of its properties, Eur. Phys. J. C 74 (2014) 3076 [arXiv:1407.0558] [INSPIRE].

[72] CMS collaboration, Search for dark matter, extra dimensions and unparticles in monojet events in proton-proton collisions at $\sqrt{s}=8$ TeV, Eur. Phys. J. C 75 (2015) 235 [arXiv: 1408.3583] [INSPIRE].

[73] ATLAS collaboration, Search for new phenomena in the dijet mass distribution using $p-p$ collision data at $\sqrt{s}=8$ TeV with the ATLAS detector, Phys. Rev. D 91 (2015) 052007 [arXiv:1407.1376] [INSPIRE].

[74] J. Jaeckel and M. Spannowsky, Probing MeV to $90 \mathrm{GeV}$ axion-like particles with LEP and LHC, Phys. Lett. B 753 (2016) 482 [arXiv:1509.00476] [InSPIRE].

[75] Fermi-LAT collaboration, W.B. Atwood et al., The Large Area Telescope on the Fermi Gamma-ray Space Telescope Mission, Astrophys. J. 697 (2009) 1071 [arXiv:0902.1089] [INSPIRE].

[76] A.A. Abdo et al., Fermi LAT Search for Photon Lines from 30 to 200 GeV and Dark Matter Implications, Phys. Rev. Lett. 104 (2010) 091302 [arXiv:1001.4836] [INSPIRE].

[77] Fermi-LAT collaboration, M. Ackermann et al., Fermi LAT Search for Dark Matter in Gamma-ray Lines and the Inclusive Photon Spectrum, Phys. Rev. D 86 (2012) 022002 [arXiv: 1205.2739] [INSPIRE].

[78] FERMI-LAT collaboration, M. Ackermann et al., Updated search for spectral lines from Galactic dark matter interactions with pass 8 data from the Fermi Large Area Telescope, Phys. Rev. D 91 (2015) 122002 [arXiv:1506.00013] [INSPIRE].

[79] Fermi-LAT collaboration, M. Ackermann et al., Constraining Dark Matter Models from a Combined Analysis of Milky Way Satellites with the Fermi Large Area Telescope, Phys. Rev. Lett. 107 (2011) 241302 [arXiv:1108.3546] [INSPIRE].

[80] FERmi-LAT collaboration, M. Ackermann et al., Dark matter constraints from observations of 25 Milky Way satellite galaxies with the Fermi Large Area Telescope, Phys. Rev. D 89 (2014) 042001 [arXiv:1310.0828] [INSPIRE].

[81] FERmi-LAT collaboration, M. Ackermann et al., Searching for Dark Matter Annihilation from Milky Way Dwarf Spheroidal Galaxies with Six Years of Fermi Large Area Telescope Data, Phys. Rev. Lett. 115 (2015) 231301 [arXiv:1503.02641] [INSPIRE].

[82] HESS collaboration, A. Abramowski et al., Search for Photon-Line-like Signatures from Dark Matter Annihilations with H.E.S.S., Phys. Rev. Lett. 110 (2013) 041301 [arXiv: 1301.1173] [INSPIRE].

[83] PAMELA collaboration, O. Adriani et al., PAMELA results on the cosmic-ray antiproton flux from $60 \mathrm{MeV}$ to $180 \mathrm{GeV}$ in kinetic energy, Phys. Rev. Lett. 105 (2010) 121101 [arXiv: 1007.0821] [INSPIRE]. 
[84] G. Bélanger, C. Boehm, M. Cirelli, J. Da Silva and A. Pukhov, PAMELA and FERMI-LAT limits on the neutralino-chargino mass degeneracy, JCAP 11 (2012) 028 [arXiv:1208.5009] [INSPIRE].

[85] AMS-02 collaboration, The AMS Experiment, talks at AMS Days at CERN, Geneva Switzerland, 15-17 April 2015.

[86] G. Giesen et al., AMS-02 antiprotons, at last! Secondary astrophysical component and immediate implications for Dark Matter, JCAP 09 (2015) 023 [arXiv:1504.04276] [INSPIRE].

[87] A. Ibarra, S. Lopez Gehler and M. Pato, Dark matter constraints from box-shaped gamma-ray features, JCAP 07 (2012) 043 [arXiv: 1205.0007] [INSPIRE].

[88] A. Ibarra, A.S. Lamperstorfer, S. López-Gehler, M. Pato and G. Bertone, On the sensitivity of CTA to gamma-ray boxes from multi-TeV dark matter, JCAP 09 (2015) 048 [arXiv: 1503.06797] [INSPIRE]. 